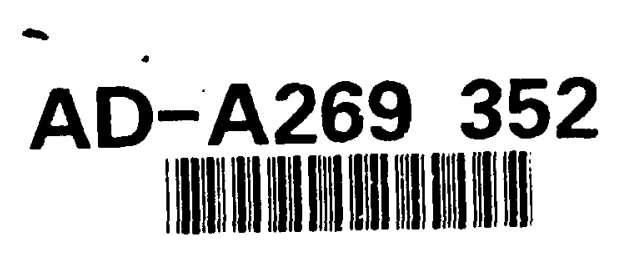

RL-TR-93-115

Final Technical Report

June 1993

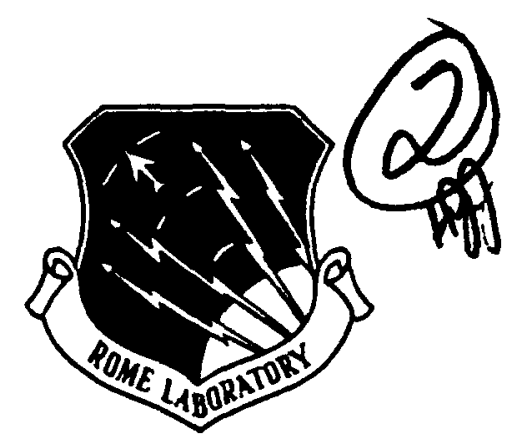

\title{
PHOTOREFRACTIVE \\ RECEIVER STUDY
}

Harris Corporation

DTIC

ELECTE SEP 151993

o

Sponsored by

Strategic Defense Initiative Office

APPAOVED FOR PUBLN RELEASE; DISTRIBUTION UNLIMITED.

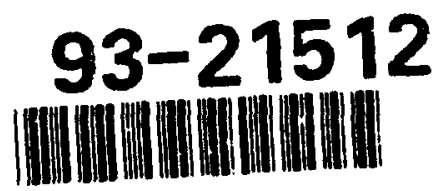

The views and conclusions contained in this document are those of the authors and should not be interpreted as necessarily representing the official policies, either expressed or implied, of the Strategic Defense Initiative Office or the U.S. Government.

Rome Laboratory

Air Force Materiel Command Griffiss Air Force Base, New York

$\begin{array}{llll}93 & 9 & 14 & 188\end{array}$ 
This report has been reviewed by the Rome Laboratory Public Affairs office (PA) and is releasable to the National Technical Information Service (NTIS). At NTIS it will be releasable to the general public, including foreign nations.

RL-TR-93-115 has been reviewed and is approved for publication.

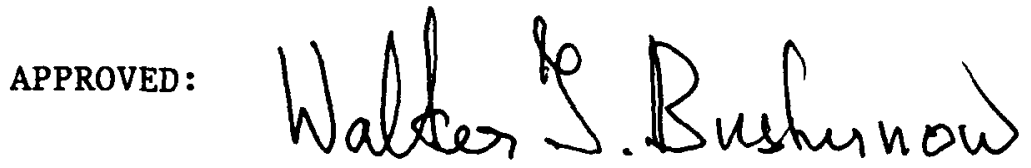

WALTER J. BUSHUNOW

Project Engineer

FOR THE COMMANDER

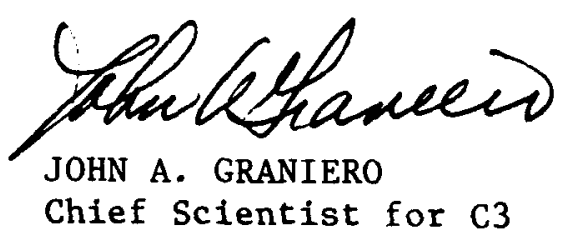

If your address has changed or if you wish to be removed from the Rome Laboratory malling list, or if the addressee is no longer employed by your organization, please notify RL ( C3BA ) Griffiss AFB NY 13441. This w111 assist us in maintaining a current mailing list.

Do not return copies of this report unless contractual obligations or notices on a specific document require that it be returned. 
M. R. Lange

D. W. Graves

Contractor: Harris Corporation

Contract Number: F30602-91-C-0129

Effective Date of Contract: 6 Sep 91

Contract Expiration Date: 18 Dec 92

Short Title of Work: Photorefractive Receiver Study

Period of Work Covered: 6 Sep 91 - 18 Dec 92

Principal Investigator: Dr. M. R. Lange

Phone: (407) 727-5119

Project Engineer: Walter J. Bushunow

Phone: (315) 330-3091

Approved for Public Release; Distribution Unlimited.

This research was supported by the Strategic Defense Inflative Office of the Department of Defense and was monitored by Walter J. Bushunow RL (C3BA), 525 Brooks Road, Griffiss AFB NY 13441-4505, under Contract F3060291-C-0129. 


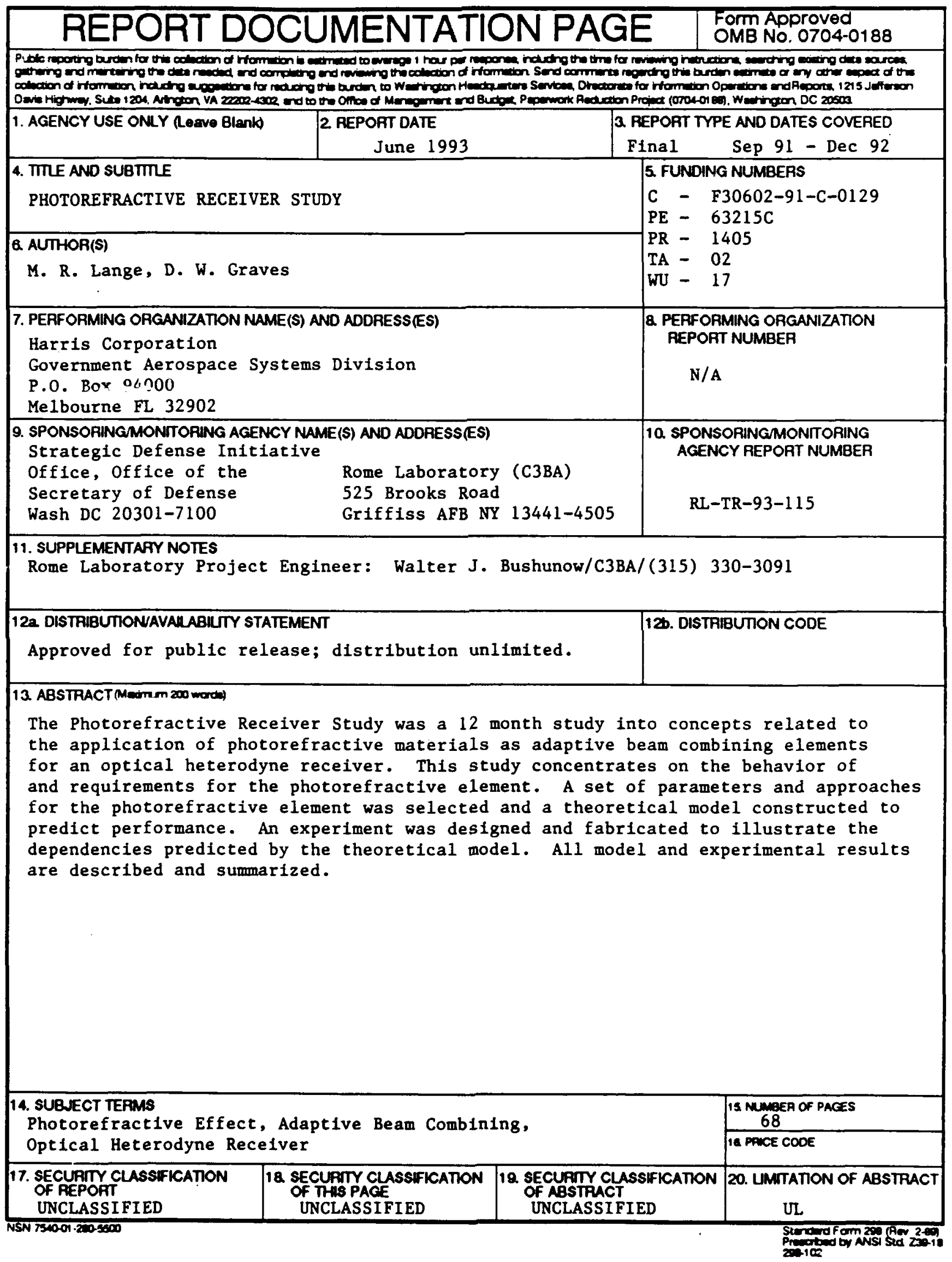


Photorefractive Heterodyne Receiver Final Report

I. Introducrion

II. Parameter study

III. Experimental results

IV. Heterodyne Gain

v. Conclusions and furure potential

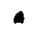

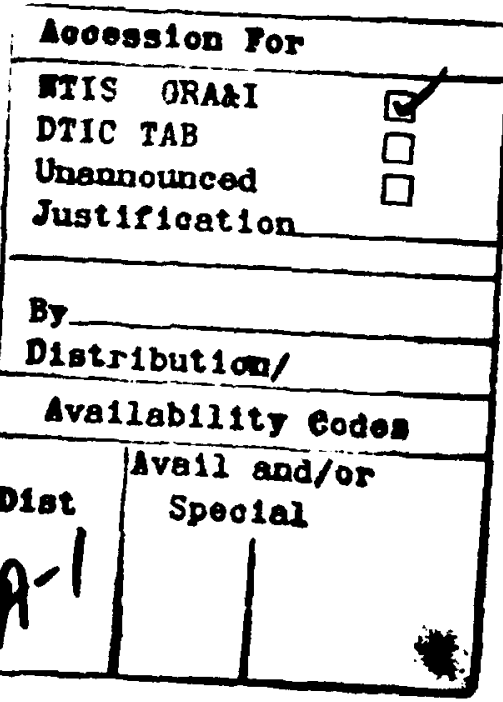




\section{TABLES}

II-1 Gain measurements

IV-1 Gain comparison

\section{FIGLRES}

III-1 Experimental layout

III-2 Optical layout

III-3 Effect of pump intensity on amplitude and bandwidth

III-4 Effect of grating wavelength and pump intensity on bandwidth

III-5 Effect of electric field strength on detected signal level

III-6 Effect of electric field strength on resonant frequency

III-7 Effect of pump intensity and grating wavelength on bandwidth

III-8 Effect of grating wavelength on peak resonant frequency

III-9 Effect of reference intensity on resonant amplirude and bandwidth

III-10 Experionental comparison with heterodyne gain

III-11 Effect of signal intensity on resonant bandwidth and amplitude

III-12 Effect of beam ratio on amplitude and bandwidth

III-13 Effect of material attenuation on resonant amplitude and bandwidth

III-14a) Detector output vs. frequency offset

III-14b) Detector output vs. vibration frequency

IV-1 Modulation techniques

IV-2 System concept

IV-3 Heterodyne gain

IV-4 Heterodyne SNR

IV-5 Heterodyne gain, shot noise limited

IV-6 Comparison of conventional to photorefractive beam combiners 


\section{Introduction}

The following report is the final report on a 12 month srudy into concepts related to the application of photorefractive materials as adaptive beam combining elements for an optical heterodyne receiver. This study concentrates on the behavior of and requirements for the photorefractive element. As such the models developed and the experiments performed are aimed at quantifying the material requirements.

The experimental parameters chosen for the study are based party on experience, a literarure search, and material and equipment availability. This study was not scoped to investigate multiple approaches. The approach chosen was determined to offer the greatest amount of insight, though not necessarily the best performance. Section II provides insight into available parameters and approaches.

The experiments performed on the study are presented in Section III. They were aimed at illustrating the parameter dependence predicted by the theoretical results presented in Section IV. They also illustrate some of the well known photorefractive parameters dependencies to establish a baseline and demonstrate the performance enhancements by optimizing these parameters. A video tape has been produced on this program illustracing one of the key advantages of the photorefractive beam combiner, plafform jitter compensation. This is discussed in Section III.

Heterodyne gain is the topic of Section IV. The modeling performed on this study is outlined in Section IV and the most significant results presented. The purpose of this section is to illustrate the requirements of the photorefractive beam combiner for receiver applications. The analysis is primarily oriented towards illustrating the advantages, disadvantages, and limitations of a photorefractive beam combiner from a systems point of view. A discussion of heterodyne reception begins below in the current section in order to lay the groundwork for the following sections.

Conclusions and recommendations are presented in the final section. There are several new developments in materials technology which open new possibilities for photorefractive elements. These are discussed and conclusions are drawn as to their potential for application to heterodyne reception.

\section{Heterodyne techniques}

Heterodyne signal reception has been a widely used concept since the early days of radio. A beterodyne receiver utilizes a local oscillator to mix with the received signal to enhance the signal reception. Optical heterodyne receivers work in a similar manner for similar reasons. At the radio carrier frequencies heterodyne reception is a common practice. 
An optical implementation of this is not common in operational systems.

There are several difficulties in implementing heterodyne techniques at oprical carrier frequencies due to the high optical frequencies (shor wavelengths). The first obvious difficulty is that oprical sources are not typically stable down to frequencies usable for signal transmission and reception. This is quickly changing, though. New solid state diode pumped lasers are commercially available with linewidths in the kiloHertz.

Given narrow line laser technology, the frequency of the transmitrer must still be racked by the receiver. This can be done by getting the signals close enough for the receiver to acquire the transmitter frequency and then track it. It may be possible to lock the transmitter and receiver to some known absolute frequency source (atomic lines uncer controlled conditions) and electronically track out residual errors that may exist.

The next difficulty encountered is in collecting the signal and combining it coherently with the oscillator. It can be difficult to collect the transmitred light and combine it coherently with a local source if it has suffered any significant phase front degradacion. One can sample smaller and smaller cross sections of light until sufficient phase front integrity is obtained at the cost of collecting less light. Or one can try 10 compensate for the phase front errors. Another difficulty related to the short wavelength is that small motion or vibration cause many wavelength shifts in position, and thus can cause noise or further demands on the frequency racking.

A significant increase in complexity of a system results from the requirements of an optical heterodyne technique, over a system that unilizes direct detect techniques. One must, therefore, ask for a clear quantification of the benefits of this increased complexity.

The performance of a receiver is limited by the signal-to-noise-ratio out of the receiver. The ultimate performance of an optical receiver is realized in the signal to shot noise received (a photon noise limited system). If the shot noise is the dominant noise term then that is the best performance that particular receiver can have for a given signal level. A large local oscillator used for heterodyne detection of a small received signal will necessarily degrade the shot noise limited signal-to-noise-ratio of the receiver output compared to a direct detect system. The primary advaniage offered by an optical heterodyne technique is in bringing a weak signal up to the shot noise limited region of the detector. That is, if the detector is, for example, thermal noise limited with the incident signal level, a sufficiently large local oscillator mixed with this signal will produce a large cross product term on the detector and bring the signal up to shot noise limited performance.

The key issues that evolved in this study relevant to the performance limitations of the photorefractive beam combiner for heterodyne reception are discussed in Section $\mathrm{V}$ below. Several of the significant issues discussed are; arbitrarily large pump sources cannot be used in a photorefractive beam combiner to increase signal gain, optical attenuation in the photorefractive material can be a severe limitation, and photorefractive gain-bandwidth is a 
critical figure of merit for a heterodyne beam combiner application.

\section{Parameter Study}

A liveranure survey of experiments conducted for photorefractive gain measurements was performed on this study. The results are presented below in tabular form. There is a large array of experimental approaches to achieving photorefractive gain in several different materials. The materials emphasized in this study are the fast response time materials, comprised mostly of semiconductors.

A detailed theory of these materials can be found in the references and will not be presented bere. The goal here is to illustrate the range of parameters achievable as determined by leading experimenters in the field. These parameters will then be used for comparison of the various approaches with respect to an optical heterodyne receiver applications.

Note in the first column of the table is a reference to the technique used to achieve photorefractive gain. These various techniques are explained in great detail in the references. so that level of explanation is not offered here. The general idea involved is that when the incident optical intensity pattern has a certain phase with respect to the forming refractive index grating the light will be preferentially diffracted along one path and the reinforcement of light intensity will cause the grating to exponentially grow along the path of light diffraction until the light in the other path that helped form the initial grating becomes depleted. Thus, the smaller the initial light component is in the preferential path, the larger the gain potential. Here gain is defined as the ratio of light level, after passing through the crystal, with and without the interferer present.

The second column lists the material band-gap for comparison with the wavelength utilized. The third column shows the time response of the interaction. Most of these are estimates based on scaling relationships and are here for reference only. Gain coefficient, gain, and loss (optical attenuation) are shown in the fourth column of the table, in addition to spatial modulation wavelength and bias voltage. The wavelength of the interaction is shown in the last column along with the beam ratio, with reference to the first author on the work

This table is not all inclusive, but a sampling of some of the better experimental measurements of gain. All of these parameters were used in the models developed below to estimate their performance as a beam combining element for a receiver application. This provides a range of potential beam combiner parameters in order to develop an understanding of potential performance that might be obtained with materials currently available. 
TABLE II-1 Gain measurements

\begin{tabular}{|c|c|c|c|c|}
\hline Material & Bandgap & Time response & $\begin{array}{l}\text { Gain Coeff. } \\
\text { loss (gain) }\end{array}$ & $\begin{array}{l}\text { Wavelength } \\
\text { Beam Ratio }\end{array}$ \\
\hline $\begin{array}{l}\text { CoTe:V } \\
\text { (AC bias) } \\
200 \mathrm{kHz}\end{array}$ & $\begin{array}{l}1.56 \mathrm{eV} \\
(0.8 \mathrm{uM})\end{array}$ & $\begin{array}{l}\text { 4. ms } \\
@ \\
1 \mathrm{~W} / \mathrm{cm} 2 \\
\text { est }\end{array}$ & $\begin{array}{c}3.3 \mathrm{~cm}-1,4 . \mathrm{mm} \\
0.8 \mathrm{~cm} \cdot 1 \quad(3.7) \\
@ \\
5.5 \mathrm{uM}, 10 \mathrm{kV} / \mathrm{cm}\end{array}$ & $\begin{array}{l}1.32 \text { uM } \\
\text { Ziari }\end{array}$ \\
\hline $\begin{array}{l}\text { GaAs:Cr } \\
\text { (moving } \\
\text { fringes) } \\
50 \mathrm{~Hz}\end{array}$ & $\begin{array}{l}1.42 \mathrm{eV} \\
(0.87 \mathrm{uM})\end{array}$ & $\begin{array}{l}\text { 40. ms } \\
@ \\
0.05 \mathrm{~W} / \mathrm{cm} 2 \\
\text { meas } \\
\end{array}$ & $\begin{array}{c}6.5 \mathrm{~cm}-1,3.6 \mathrm{~mm} \\
1.65 \mathrm{~cm}-1 \quad(10) \\
@ \\
18 \mathrm{uM}, 8.6 \mathrm{kV} / \mathrm{cm}\end{array}$ & $\begin{array}{l}1.064 \mathrm{uM} \\
1000 / \mathrm{i} \\
\text { Imbert }\end{array}$ \\
\hline $\begin{array}{l}\text { InGaAs:El2 } \\
\text { (Franz-Keldyshi } \\
\text { moving fringe) } \\
4 \mathrm{~Hz}\end{array}$ & $\begin{array}{l}1.42 \mathrm{eV} \\
(0.87 \mathrm{uM})\end{array}$ & $\begin{array}{l}\text { 340. ms } \\
@ \\
0.140 \mathrm{~W} / \mathrm{cm} 2 \\
\text { est }\end{array}$ & $\begin{array}{c}16.3 \mathrm{~cm}-\mathrm{i}, 4 . \mathrm{min} \\
3 \mathrm{~cm}-1 \quad(600) \\
@ \\
7 \mathrm{uM}, 10 \mathrm{kV} / \mathrm{cm}\end{array}$ & $\begin{array}{c}0.94 \text { uM } \\
4800 / 1 \\
\text { Partovi }\end{array}$ \\
\hline $\begin{array}{l}\text { GaAs:El2 } \\
\text { (trap } \\
\text { saturation) }\end{array}$ & $\begin{array}{l}1.42 \mathrm{eV} \\
(0.87 \mathrm{uM})\end{array}$ & $\begin{array}{c}\text { 30. ms } \\
@ \\
0.013 W / c m 2 \\
\text { est }\end{array}$ & $\begin{array}{c}2.6 \mathrm{~cm}-1,6 . \mathrm{mm} \\
1.3 \mathrm{~cm}-1 \quad(4.7) \\
@ \\
9 u M, 13 \mathrm{kV} / \mathrm{cm}\end{array}$ & $\begin{array}{l}1.064 \text { uM } \\
60 / 1 \\
\text { Duncan }\end{array}$ \\
\hline $\begin{array}{c}\text { GaAs:El2 } \\
\text { (AC bias) } \\
20 \mathrm{kHz}\end{array}$ & $\begin{array}{l}1.42 \mathrm{eV} \\
(0.87 \cup M)\end{array}$ & $\begin{array}{c}\text { 10. ms } \\
@ \\
0.02 \underset{\text { Walcm }}{\mathrm{W} / \mathrm{cm}} \\
\end{array}$ & $\begin{array}{l}2.4 \mathrm{~cm}-1,10 . \mathrm{mm} \\
0.87 \mathrm{~cm}-1(11) \\
@ \\
15 \mathrm{uM}, 3.2 \mathrm{kV} / \mathrm{cm} \\
\end{array}$ & $\begin{array}{l}1.064 \text { uM } \\
10 \text { to } 1000 ; 1 \\
\text { Klein }\end{array}$ \\
\hline $\begin{array}{l}\text { InP:Fe } \\
\text { (trap } \\
\text { saturation) }\end{array}$ & $\begin{array}{l}1.35 \mathrm{eV} \\
(0.92 \mathrm{uM})\end{array}$ & $\begin{array}{l}30 \mathrm{~ms} \\
@ \\
0.03 \mathrm{~W} / \mathrm{cm} 2 \\
\text { meas }\end{array}$ & $\begin{array}{l}\text { 4. } \mathrm{cm}-1,4 \mathrm{~mm} \\
\text { 2. } \mathrm{cm}-1 \quad(5) \\
@ \\
5 u M, 8 \mathrm{kV} / \mathrm{cm}\end{array}$ & $\begin{array}{l}1.064 \text { uM } \\
80 / 1 \\
\text { Mainguet }\end{array}$ \\
\hline $\begin{array}{l}\text { InP:Fe } \\
\text { (thermal } \\
\text { balance) } \\
\text { (grad) } \\
\end{array}$ & $\begin{array}{l}1.35 \mathrm{eV} \\
(0.92 \mathrm{uM})\end{array}$ & $\begin{array}{l}\text { 150. ms } \\
@ \\
0.009 \text { W:sm2 } \\
\text { est }\end{array}$ & $\begin{array}{r}6.5 \mathrm{~cm}-1,7.2 \mathrm{~mm} \\
2.6 \mathrm{~cm}-1 \text { (100) } \\
\text { @uM, 10kV/cm }\end{array}$ & $\begin{array}{c}1.064 \text { uM } \\
8000 / 1 \\
\text { Ozkul }\end{array}$ \\
\hline $\begin{array}{l}\text { InP:Fe } \\
\text { (incoherent } \\
\text { backlight) }\end{array}$ & $\begin{array}{l}1.35 \mathrm{eV} \\
(0.92 \mathrm{uM})\end{array}$ & $\begin{array}{l}60 . \mathrm{ms} \\
@ \\
0.015 \mathrm{~W} / \mathrm{cm} 2 \\
\text { est }\end{array}$ & 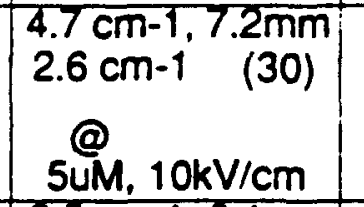 & $\begin{array}{c}1.064 \text { uM } \\
1500 / 1 \\
\text { Ozkul }\end{array}$ \\
\hline $\begin{array}{l}\text { InP:Fe } \\
\text { (AC bias) } \\
400 \mathrm{~Hz}\end{array}$ & $\begin{array}{l}1.35 \mathrm{eV} \\
(0.92 \mathrm{uM})\end{array}$ & $\begin{array}{c}0.58 \mathrm{~ms} \\
@ \\
0.03 \mathrm{~W} / \mathrm{cm} 2 \\
\text { meas }\end{array}$ & $\begin{array}{c}2.5 \mathrm{~cm}-1,9.1 \mathrm{~mm} \\
0.15 \mathrm{~cm}-1 \quad(9.7) \\
@ \\
25 \mathrm{uM}, 10 \mathrm{kV} / \mathrm{cm}\end{array}$ & $\begin{array}{c}1.32 u M \\
80 / 1 \\
\text { Bylsma }\end{array}$ \\
\hline
\end{tabular}




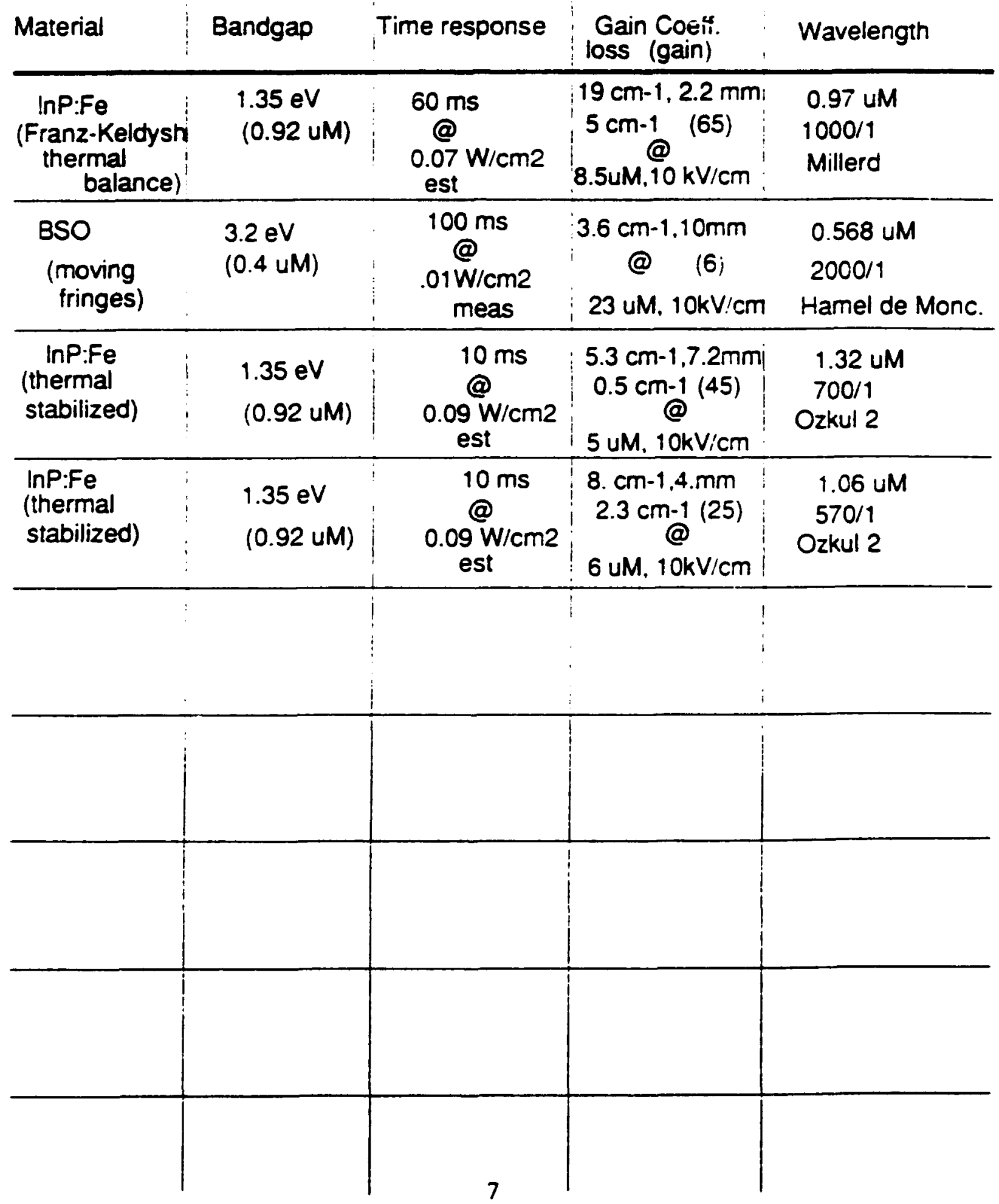




\section{Experimental Results}

\section{Experimental Background}

The juearire search xenconed above revealed severai ways ci enhancing :te photorsiacive gain in semiconductor materials used by othe: researchers. Because ai ine small electro-optc coefficient in the faster response time maierials application of an $=x: a n a:$ elecric field across the photorefracive crystal is a common pracice to enhance protoretracjue afects. When the external electic field remains constant the photoretractive grating within the crystal drifts, due to dynamic charge migrasion, along the tirecticr of its electric äeld. This drift can be adjusted to coincide with the drift of the opticai irisges produced from sxo optical beams offset from each other by a small trequency direreace. When these coincide, a 90 shift occurs between the optical fringe pattern and the photoreiracive graing. The light interference then reinforces the charge grating in a positive feedback mode and exponential growrh of the gracing intensity results. The photoreractive effect is maximized and one of the light paths will experience gain.

Two coherent beams offset by a small frequency shift can be easily created with a single laser and several acousto-optic Bragg cells. The furst Bragg cell, driven by a iriquency $W_{1}$, separates the laser beam into two beams. The undiffracted light serves as the pump beam, while the diffracted light will be further difiracled by the second Bragg cell to contain 3 modulated signal for detection. The undiffracted beam can be set several orders of magnitude stronger than the diffracted beam. The frequency of the diffracted beam has been Doppler shifted by the Bragg cell drive frequency, and is represented by $W+W_{1}$. The second Brgg cell adds a modulated signal to the beam. The Bragg cell Doppler shifis the light by its drive frequency $W_{2}$, resulting in a frequency of the beam of $W+W_{1}-W_{2}$. The negarive of the second Doppler shift term comes from Doppler shifing the optical beam down in frequency by the incidence of light in the direction of the acoustic wave. When the two frequencies $w_{1}$ and $w_{2}$ are equal then the resulting beam is perfectly phase coherent with the pump beam. A net shift in frequency occurs when the two frequencies are offset by a smail amount and the modulated beam is then shifted in frequency relative to the pump beam. If this beam interferes with the pump beam the resuling light fringe will move spatially at a velocity proportional to the frequency difference between the two beams.

The modulated signal in the second Bragg cell can be simulated in a number of ways using two frequencies present in the cell simultaneously. Two frequencies summed together produces a single sideband modulated tone. Mixing the two frequencies together generates a double sideband signal with the carrier frequency amplitude determined by the voltage offset of the modulating component. We have chosen to use i. single sideband modulation scheme in the experiment for simplicity. One frequency is nearly idencical in frequency to the frequency driving the first cell, and the second frequency, offset by about $150 \mathrm{KHz}$. represents the signal sideband containing the modulation information. These two signals, referred to as the reference and signal beams respectively, interfere at the detector to produce 
a $150 \mathrm{KHz}$ :ore whose stength is determined by the oross produci iern of the individuai amplitudes. This signal will be detected regardless of any photorefractive processes or acix thereof. The goal of the experiment is to interact the reference beam with the pump beam in the photorefracive crystal, deflecting some of the pump beam into the reference beam. This will increase the amplitude of the reference beam and the sross product term the detec:or for a heterodyne detection gain.

Estabiisining and verifying a number of relationships upon which the photoretracive gain is dependent comprised the bulk of the experimental work. Pump intensity, reference intensity, and signal beam intensity and the relative intensities between the three beams all determine the strength and bandwidth of the photorefractive gain resonance. Additionally the gain is related :o the angle of incidence between the reference and pump beams, electric field applied to the rrystal and the frequency offset between the reterence and pump beams. Other aspects of the photorefractive process such as vibration and aberration correction and resonance bandwidth were investigated.

\section{Experimental Test Setup}

Figure III- 1 shows a blor:k diagram of the experimental setup. Three signal generators, operating off a common clock to ensure relative stability, drive the two Bragg cells. Signal generator 1 drives Bragg cell 1 , used to separate the reference and signal beams from the pimp beam. Signal generators 2 and 3 drive Bragg cell 2, simulating a single side band modulated optical beam. Generator 2 creates an optical beam we call the reference, and generator 3 creates the signal beam. The output of generators 2 and 3 are summed together before entering the Bragg cell. The optical beams interact in the photorefractive crystal and a In:GaAs detector collects the resulting intensity modulated signal which is fed into a spectrum analyzer. The computer records the peak measured by the spectrum analyzer in a data file.

Figure III-2 shows a more detailed layout of the optical system. We used a Nid:Y.AC ( $1.06 \mathrm{um}$ ) laser because of its stability and very narrow line width. The only laser available during these experiments at 1.3 microns was a laser diode. This laser would not give a large enough coherence length (approximately $0.3 \mathrm{~mm}$ ) to operate a dual leg interferometer. All data presented in this report were taken at 1.06 microns.

To obtain a large enough deflection angle at $70 \mathrm{MHz}$ to sufficiently separate the pump beam we used a shear $\mathrm{TeO}_{2}$ cell for Bragg cell 1. The polarized light of the laser rotates in this cell due to the optical activity of $\mathrm{TeO}_{2}$ in the optical direction. This angle of rotation is sensitive to temperature and thus to drive power. A linear polarizer placed immediately following the Bragg cell ensures the polarization of all optical beams in the $\left[\begin{array}{lll}1 & 1 & 0\end{array}\right]$ plane of the photorefractive crystal. Since the polarization of the optical beam is sensitive to Bragg cell temperature, the resulting optical intensities were sensitive as well, and somerimes not repeatable. Standard operating practice was to tum on the Bragg cell well before taking any data and make no adjustments in drive power to the cell thereafter. 

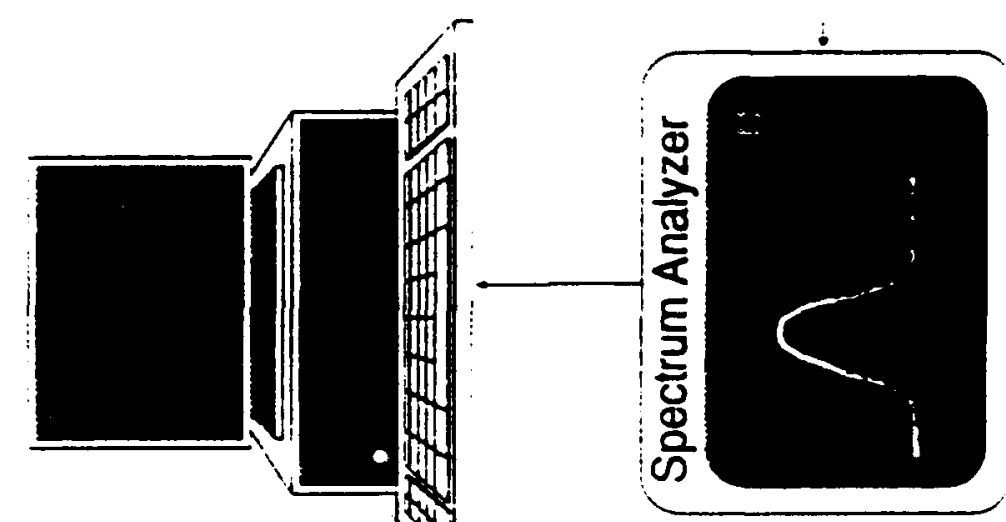

ב⿳亠二口犬

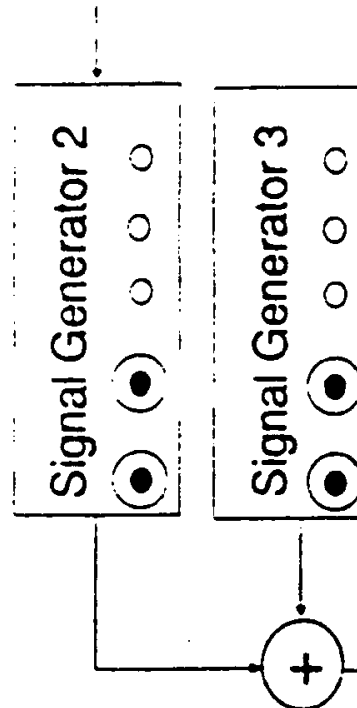



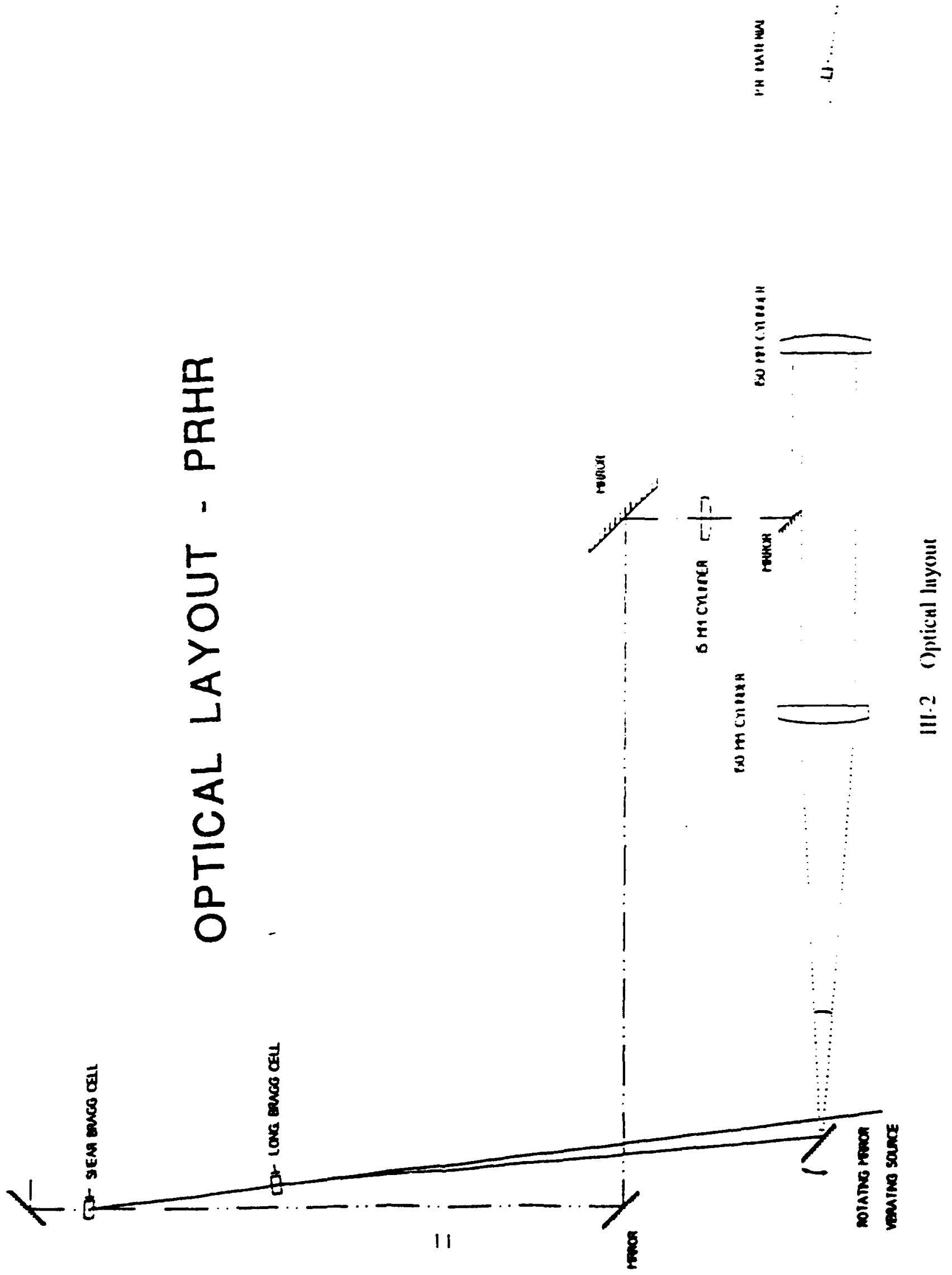


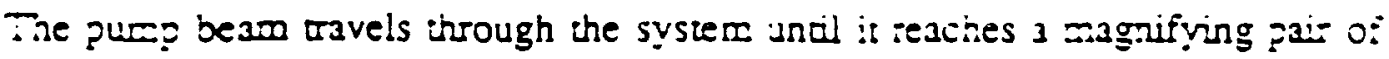
$\because \because$ lindrical lenses that increased the beam size $10 \mathrm{x}$ in the horizonul direcion betore sater:a

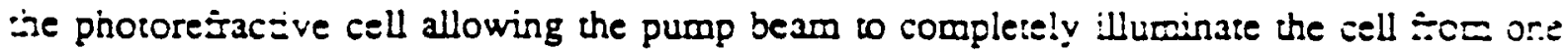
elecsode to the othe: thus limiting the possibility of shadowing effects. The reieresce 3 . $C$ signal beams ar generated with a longitudinal $\mathrm{TeO}_{2}$ cell (no optical activity). A Ei-sor :-3: zan be either rotated to provide variable incidence angles at the photorstractive se:.. ar vibrated to stucy the vibraion and aberration compensation of the system, folds the secicai path to follow ise optical table. The reference and signal beams then pass ibrough sxo cylindrical lenses that provide a $1 \mathrm{x}$ magnification in the spot size. The magnifying !enses ( $\mathrm{x}$ For the reference $30 d$ signal and $10 x$ for the pumpi share a common Fourier plane $: 2 x$ inch 2 colding niror is placed in the pump beam. This allows ine reierence and signal to pass inrough the phctoretactive crystal at small angles relasive :o pump, about 2 degras. A Letector is placed beyond the photorefracive crysial to detec: the intersity aodulazcr. produced by the ross product of the reference and signal beams.

\section{Single carrier model}

Experizents performed with this setup were designed :o measure and veffy scese of the theorejcal zeceiver calculations described in Section $N$ of this repor. In addision, some of the common Deasurements made on photorefractive materials were made to verify a baseline of initial assumptions about the material. The basis of the photorefacave calculasions coces from several equations governing the behavior of single carrier photorefractive crystals. Although it has been shown that a two carrier model is required to describe the besavior of photorefractive InP, the single carrier model (Slupeos! is suficient to explain zends in many limits. These equations are described here with appication to the experisents to describe general tends observed. not to quantify the data. Veriñcaion of assumptions of the heterodyne receiver model of Section $N$ were also made.

The magnitude of light diffraction efficiency due to the index grating is

$$
\eta \propto\left(\frac{1}{2} n^{3} r \frac{E_{\alpha}}{\sigma_{\alpha}}\right)^{2}
$$

in the small difaction limit where $n$ is the applicable refractive index and $r_{e r}$ is the effective electro-opic coefficient. This proportionality is independent of grating phase in the small diffacion limic The static index grating is generally produced by a space charge electric field developed due to charge separation, and is given by, 


$$
E_{x=}=-\frac{m E}{1-K^{2} l_{3}^{2}-i K I_{B}}
$$

in the applicabie limits where diffusion effecis are small compared to charge dift Sor the bias seld The material response time, ignoring diffusion effects, is given by the scuation.

$$
5_{x}=\frac{={ }_{w}\left(K^{2} r_{D}^{2}\right)}{\left(1-K^{2} r_{D}^{2}\right)\left(1-K^{2} l_{3}^{2}\right)+K^{2} r_{z}^{\prime} z}
$$

where,

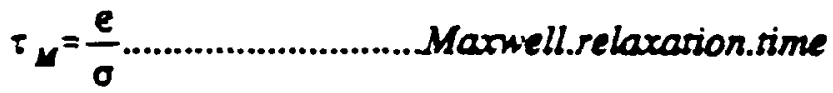

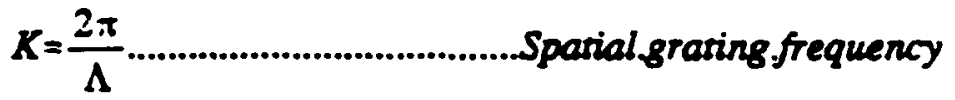

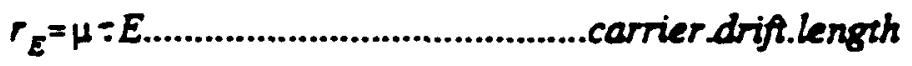

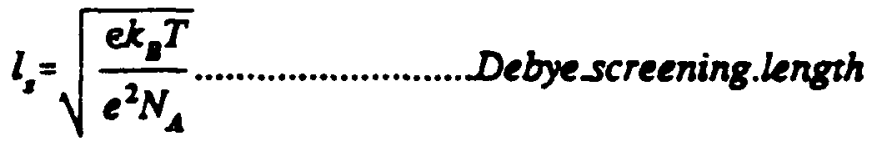

$$
\begin{aligned}
& l_{E}=\frac{e E}{e N_{A}} \text {............electron.tightenizg.length }
\end{aligned}
$$

and the notation and definitions are from Stepanov et. al. In many cases of interest the Debye screening length $l$, can be neglected. Trap saturation in the absence of an applied electric field is uncommon under usual experimental conditions.

By offsetting the reference and pump beam frequencies the grating can be made to 
drift along the s!ectric field and the maximum space charge fie!d becomes

$$
E_{x}=\frac{-i m E K r_{E}}{1+K^{2} r_{D}^{2}-K^{2} r_{E} t_{E}}
$$

At resonant frequency

$$
v_{0}=\frac{r_{E}-l_{E}\left(1+K^{2} r_{D}^{2}\right)}{r_{M}\left(K^{2} r_{D}^{2}\right)}
$$

These equations illustrate the response of a single carrier photorefractive crystal for various electric fields, pump intensities, and reference beam - pump beam relative incident angles. The resonant velocity is dependent on electric field, relative angle between beams, and pump beam intensity.

The experimental setup has the capability of independently adjusting the reference beam intensit: signal beam intensity, pump beam intensity, electric field applied to the photorefractive crystal, angle of incidence of the reference and signal beams relative to the pump beam, and the frequency offset between the reference and pump beam. In addition, three different samples of indium phosphide were used to compare various absorption coefficients within the crystals. This section presents our findings on the impact of each individual variable on the operation of the system for the conditions described.

A typical 'dataset' is collected as follows: The signal generator 1 is preset to 70 MHz. Signal generator 2 is set to $70 \mathrm{MHz}$ and signal generator 3 is set to $69.85 \mathrm{MHz}$. Once the spectrum analyzer has locked on to the $150 \mathrm{KHz}$ beat between the reference and signal beams, the computer changes signal generator 2 to a different frequency several $\mathrm{KHz}$ off 70 MHz. Typical offsets are 2 to $6 \mathrm{KHz}$. The computer then waits for the spectrum analyzer to obtain and messure the shifted signal. Once the spectrum analyzer reports a data point to the computer the signal generator is then stepped in frequency by a small amount and the computer again waits for the spectrum analyzer to complete its measurement. The computer tracks the frequency of signal generator 2 and the peak value of the signal from the spectrum analyzer and stores this information on a file from which we can construct signal versus 
frequency offset plots.

After assuring ourselves that the data we were taking were accurate and meaningful we began investigating the many variables built into the system to understand the relationship between the gain of the system and/or the bandwidth, and the heterodyne gain.

\section{Pump beam intensity}

The pump beam is typically several orders of magnitude more intense than the other beams, and hence dominates the number of photoelectrons within the crystal. The Maxwell relaxation time is inversely proportional to the photoelectron density, which in turn is proportional to pump intensity and absorption rate. The response time of the grating is directly related to $\tau_{M}$, and thus we should expect to see a faster response time as the pump intensity increases. The resonant velocity on the other hand is inversely proporional to $\tau_{M}$, and we would then expect to see the resonant velocity increase with pump power.

Figure III-3 is a composite plot of several data sets taken with different pump intensities. Each curve shows the shape of the resonance produced with the pump intensity adjusted to the amount annotated on the graph. The abscissa is plotred as frequency offset (between reference and pump beams), which is simply $K v_{0}$, the product of the grating vector and the velocity. The ordinate represents the peak signal intensity detected by the photodetector and measured by the specorum analyzer. The response time of the grating can be determined from the plots by noting that the response time is the inverse of the bandwidth. The trends apparent in this figure are that the bandwidth decreases with decreasing pump intensity and the resonant velocity decreases with decreasing pump intensity. This is consistent with predictions. Note that the signal output does not scale as the pump intensity. This is as predicted by the model in the following section. This behavior is quite different from an optical heterodyne receiver that urilizes a conventional beam combiner. This is discussed further in Section IV. Figure III-4 shows another plot of the bandwidth dependence. The different curves represent data taken with various grating wavelengths.

\section{Electric field}

The elecuric field manifests itself in the equations through the drift length $r_{E}$, and the electron tightening length $l_{E}$, as well as being explicitly found in the amplitude equation. $E_{\infty}$ is to first order proportional to the electric field applied. As the saturation point is reached, $K^{2} r_{E} l_{E}$ dominates the denominator and we find the grating amplitude to be essentially independent of electric field. Figure III-5 shows the peak signal plotted versus electric field strength. An increase in signal strength versus electric field strength is shown up to the maximum voltage of 3200 volts. This demonstrates that the photorefractive devices are operated in the non-saturated mode.

The resonant velocity should decrease with increasing electric field since $E$ is dominant in the denominator. Figure III-6 shows a plot of how the applied electric field 


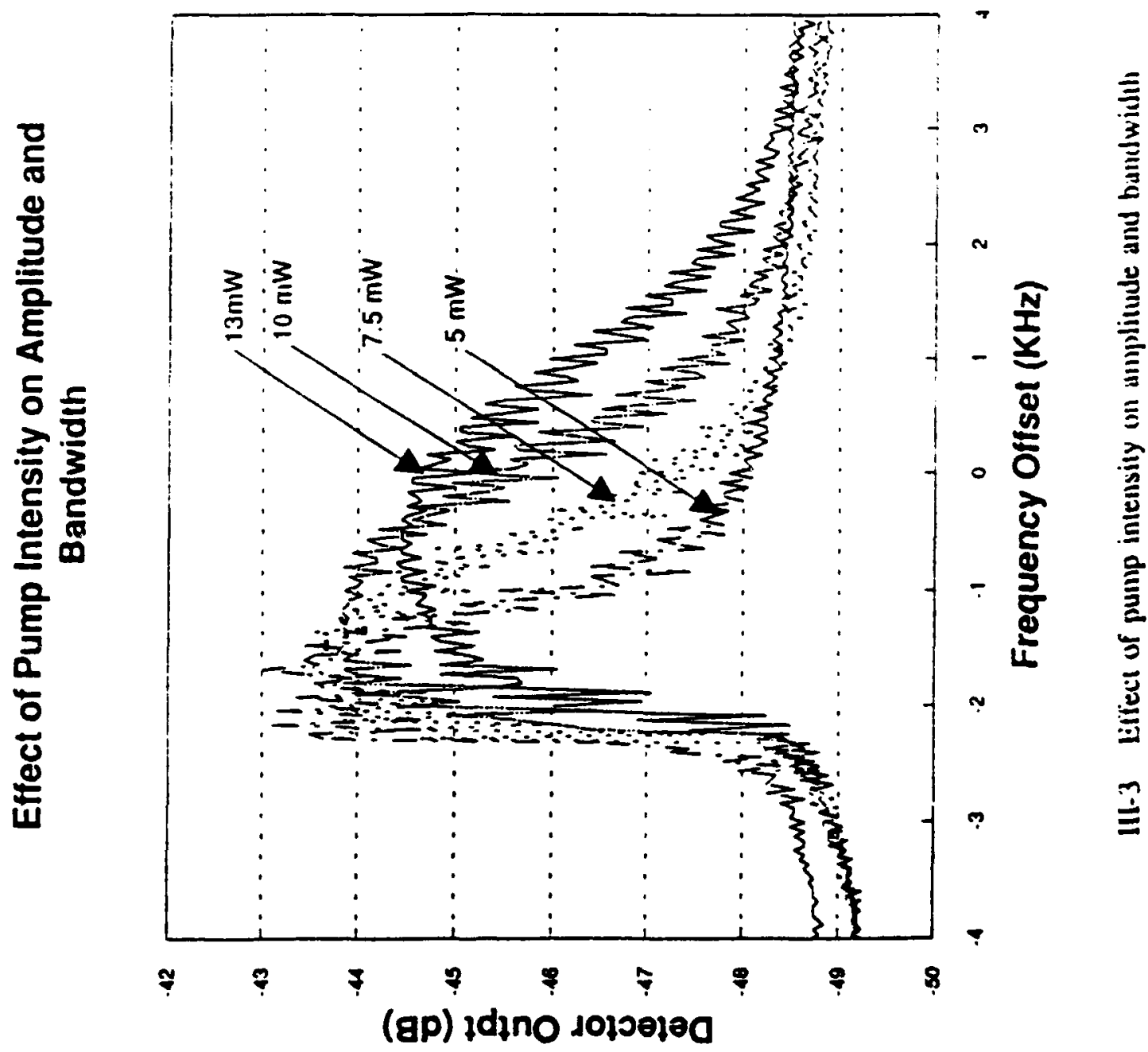




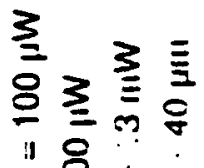

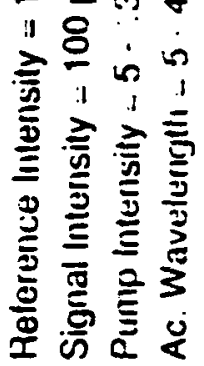

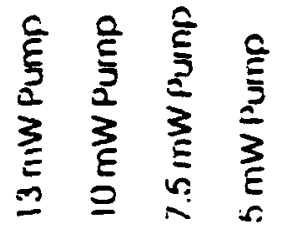

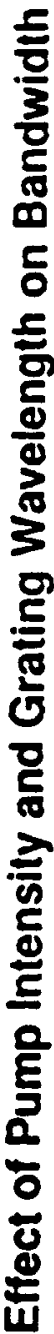
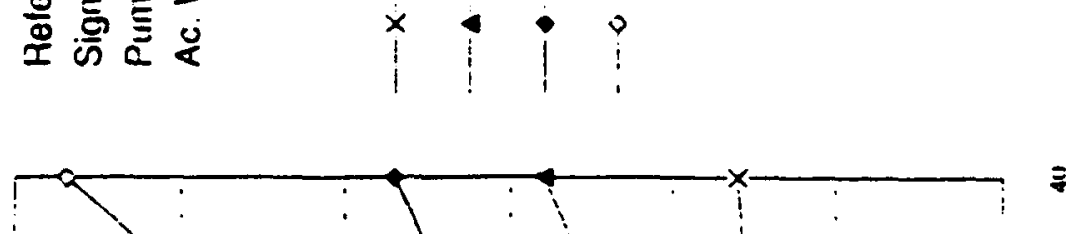

5

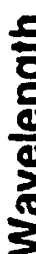

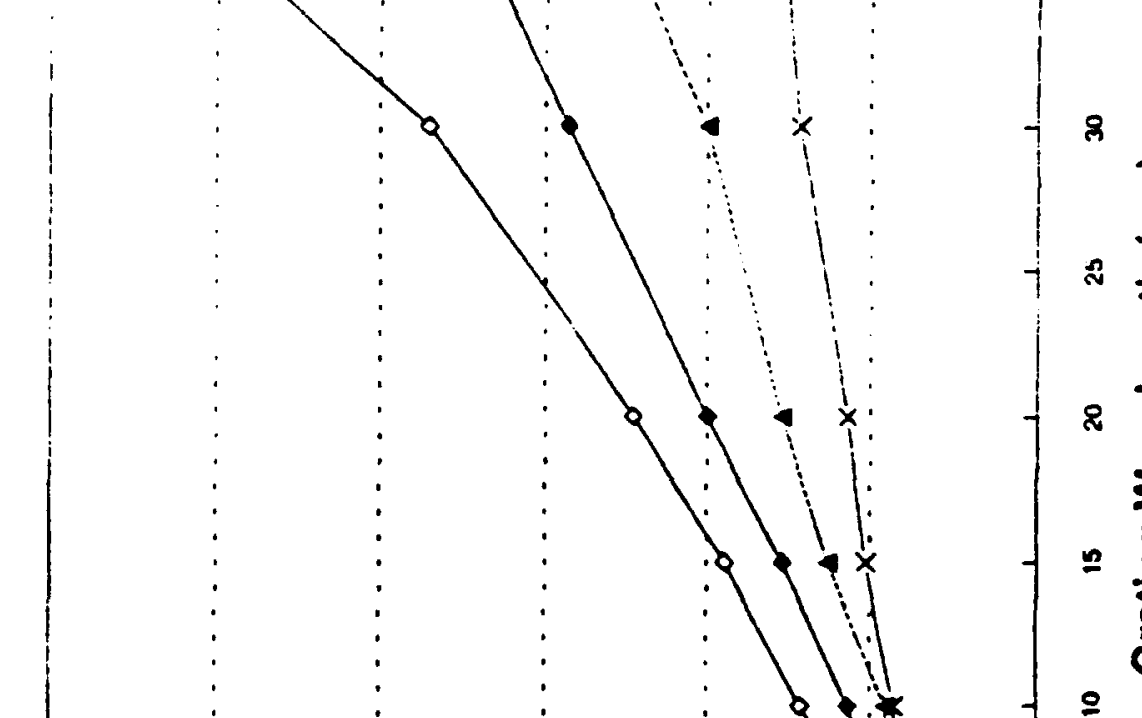

은

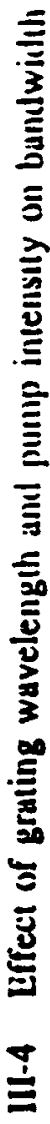

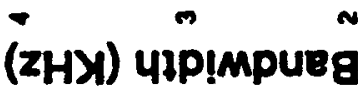




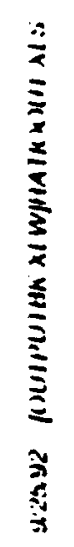

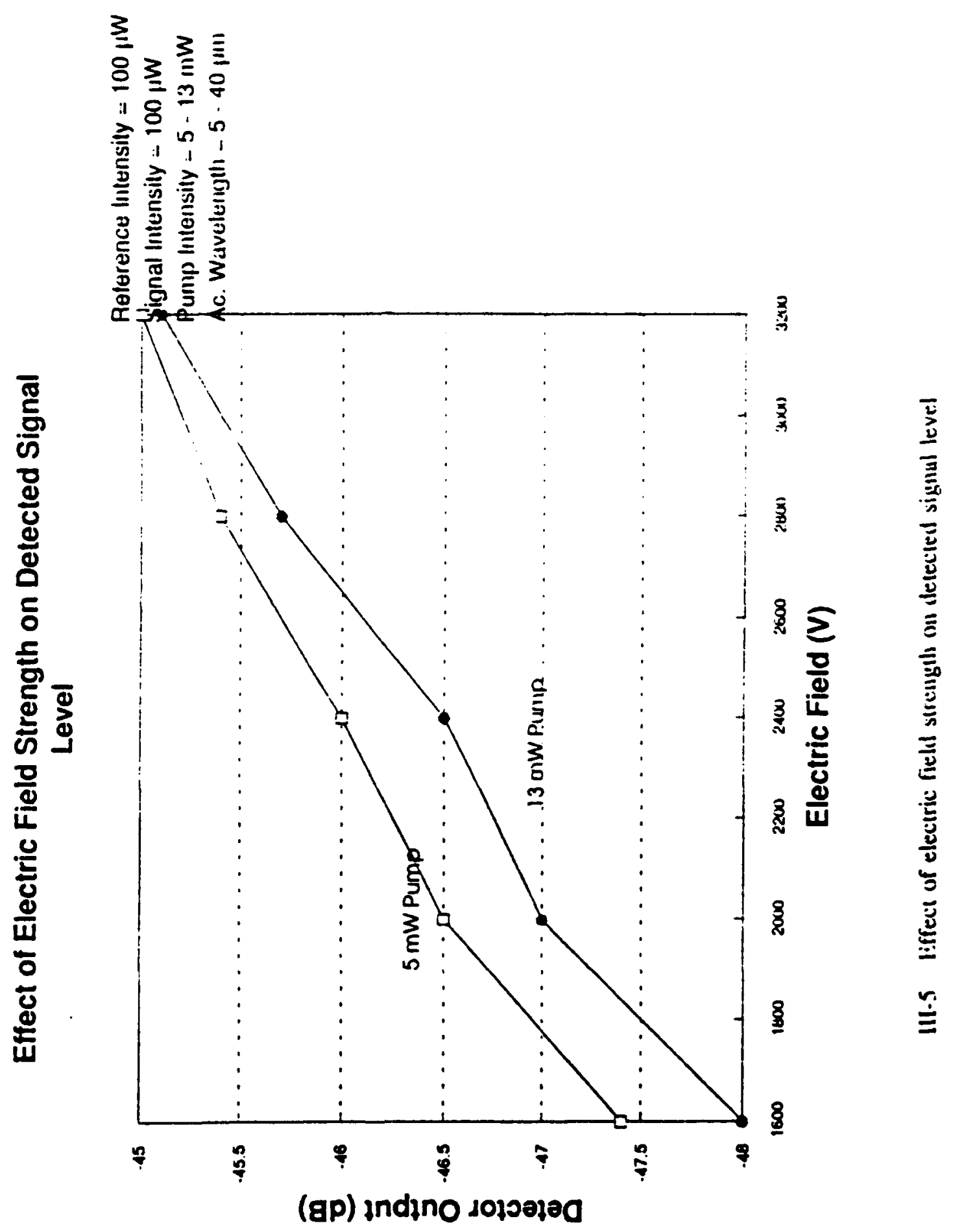




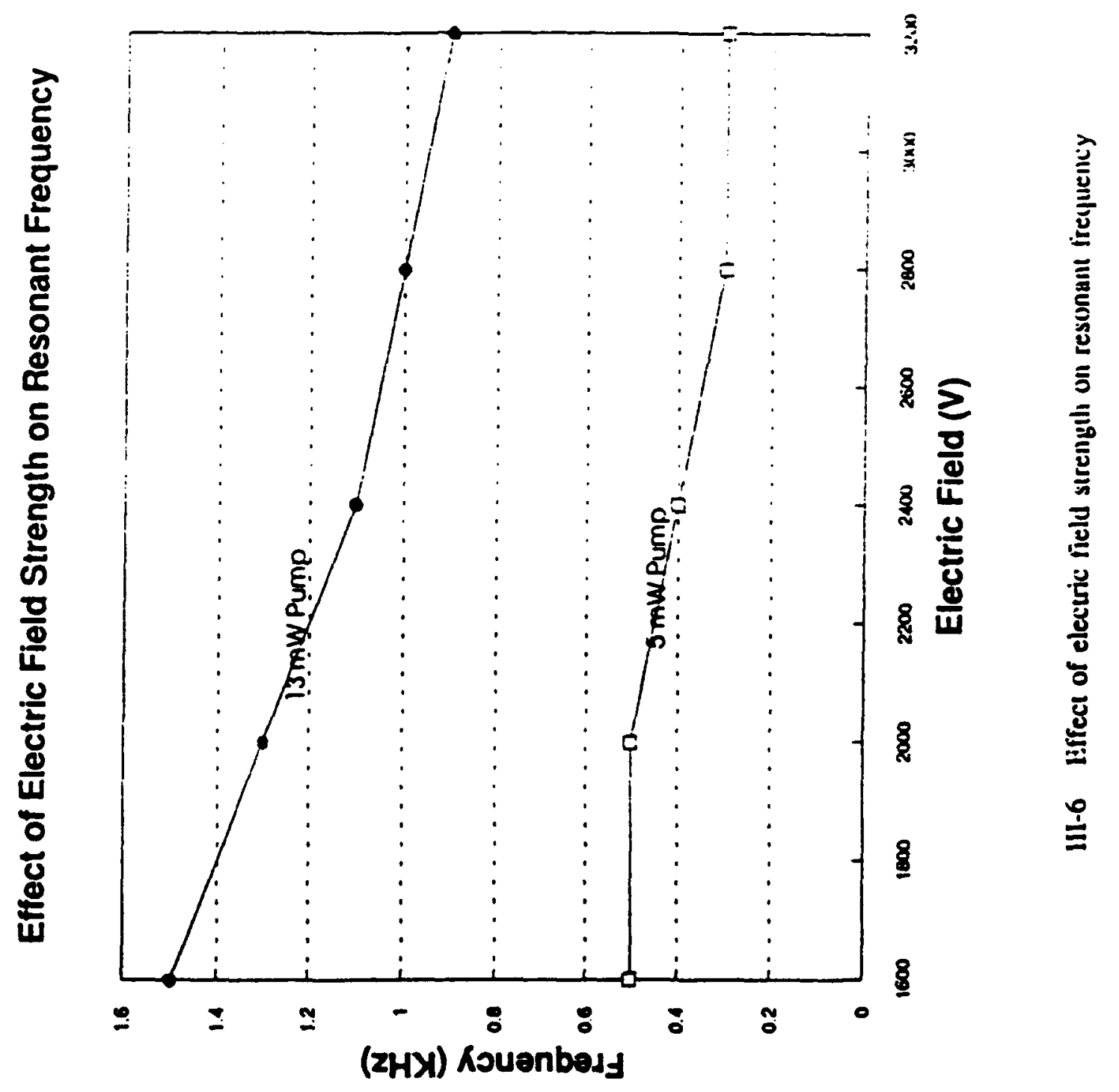


ieiates to the resonant velocity. Two curves plotted tor two different pump intensices show similar responses.

\section{Grating wavelength}

The spatial frequency of the grating shows up in both the numerator and denominator of the space charge electric field equation in a form that implies an optimum value. This is 3 commonly characterized parameter in photorefractive materials. In or near sanuration the $\mathrm{K}^{2}$ term dominates the denominator and a net inverse relationship occurs between grating amplitude and spatial frequency. The resonant velocity likewise should increase as the grating wavelength increases. Figure $\mathrm{MI}-7$ shows the bandwidth of the resonance vs. grating wavelength for various pump intensities. The data shown here is a different representation of the data contained in Figure III-4. All curves show a general increase in bandwidth as the grating wavelength increases, with the higher pump intensities providing the most extreme change. Figure III-8 shows the resonant frequency as the grating wavelength increases. Three curves are shown representing different pump intensities.

\section{Reference dependence}

Figure II-9 illustrates the relationship of the reference lightt level for all other parameters held fixed. The direct detect level drops $6 \mathrm{~dB}$ for $6 \mathrm{~dB}$ drop in reference level. The heterodyne signal follows this same general trend, but illustrates a saturation at the higher reference level. At the upper reference level the beam ratio is $12.5 / 1$. This saturation effect is illustrated in the model in the next section and is due to the fact that a small allows the diffraction efficiency to become large enough that the signal beam starts to deflect into the wrong path. This is discussed in more detail in the following section. Figure III-10 illustrates the experimental points (triangle and square) plotted against the model. The lowe: curve represents the upper trace on Figure III-9 with the smallest pump to reference beam ratio. An independent measurement determined the gain coefficient to be $1.5 \mathrm{~cm}^{-1}$ under similar conditions of illumination.

\section{Signal dependence}

The signal level dependence in Figure III-11 follows the general trend of the model predictions in that it is $\mathrm{a} \mathrm{dB}$ for $\mathrm{dB}$ dependence. The heterodyne signal follows this dependence quite well. The direct detect level shows an apparent compression at the low signal end. The low end measurement is limited by detector noise.

\section{Signal and reference dependence}

As one might expect when the received light is reduced, that is the signal and the reference component, the detector output falls $2 \mathrm{~dB}$ for every $\mathrm{dB}$ loss in the received light. 


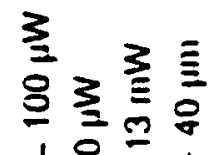

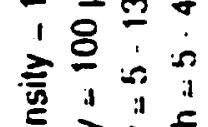

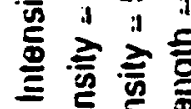

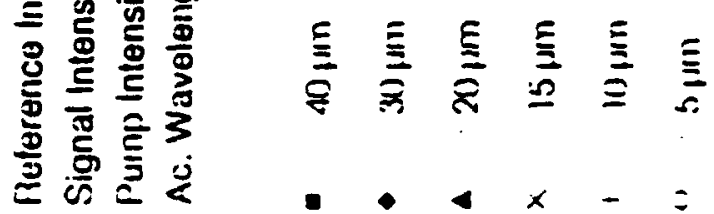

- $4 x-=$

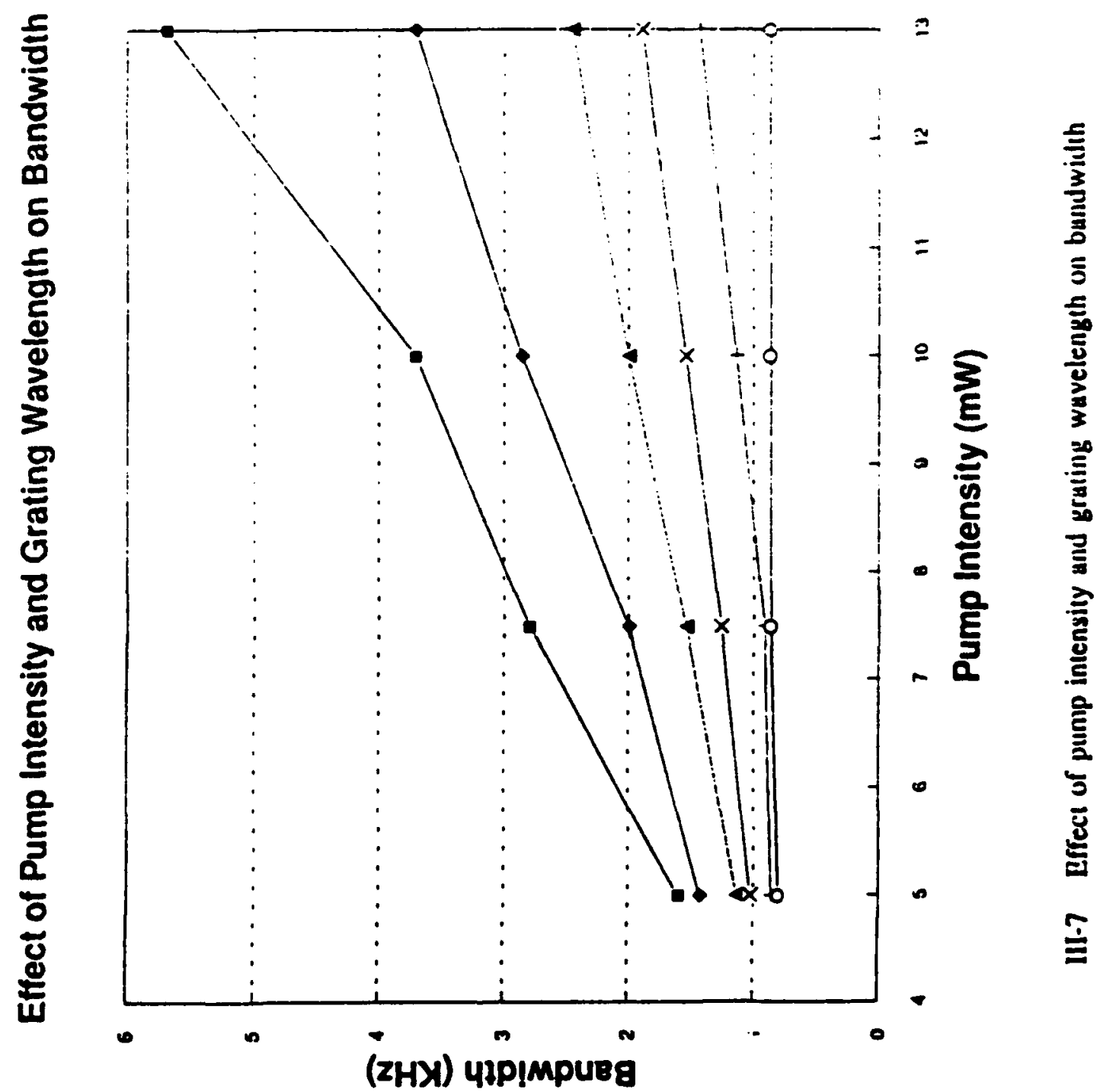




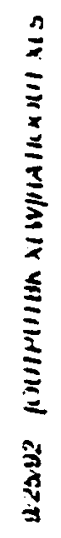

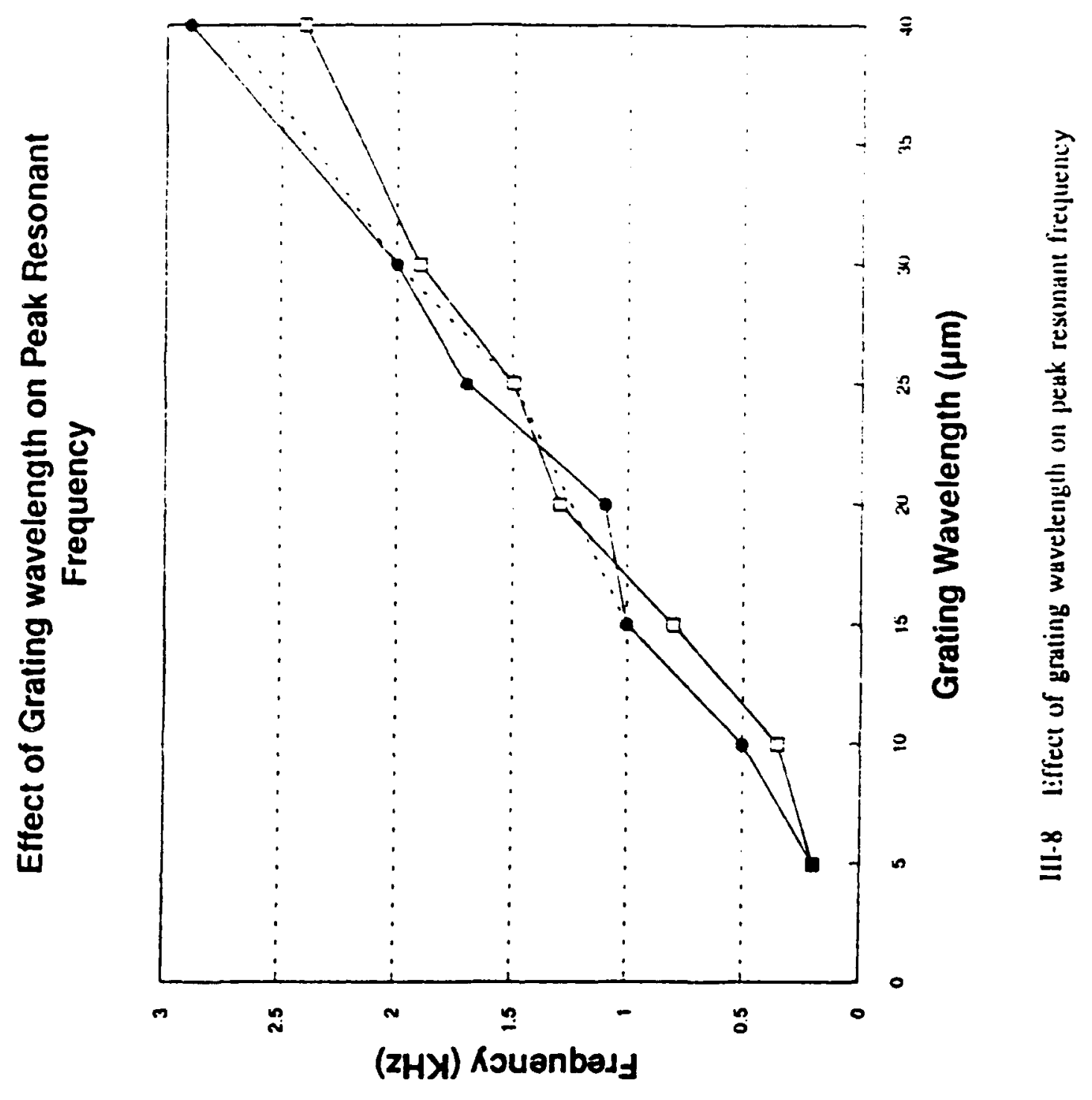



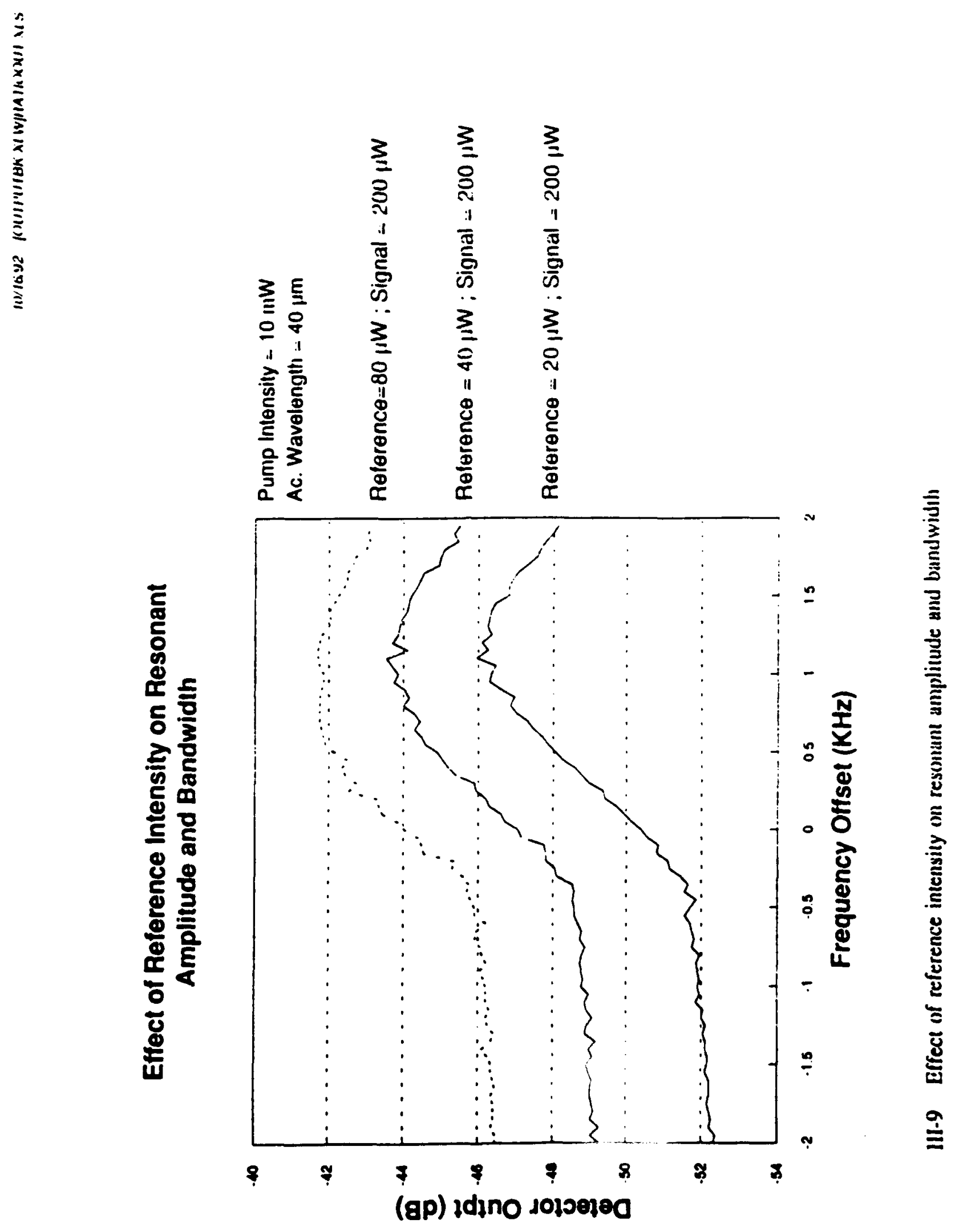


\section{Experimental comparison with Heterodyne Gain}

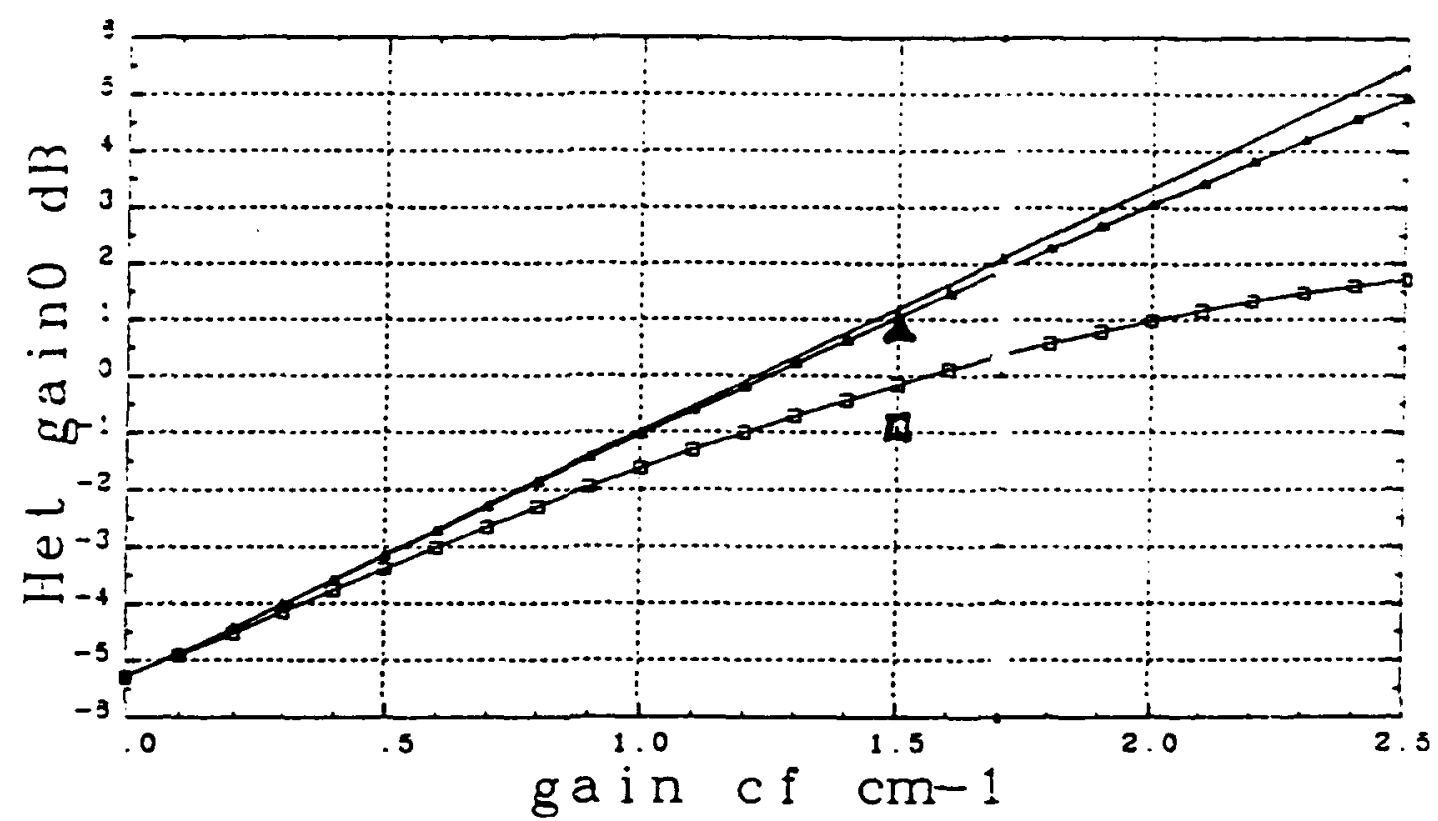

Hot $20122 \leqslant 3$

experiment gais vo gain coel. bear ratio

bel/dir gain

III-10 Experimental comparison with heterodyne gain 


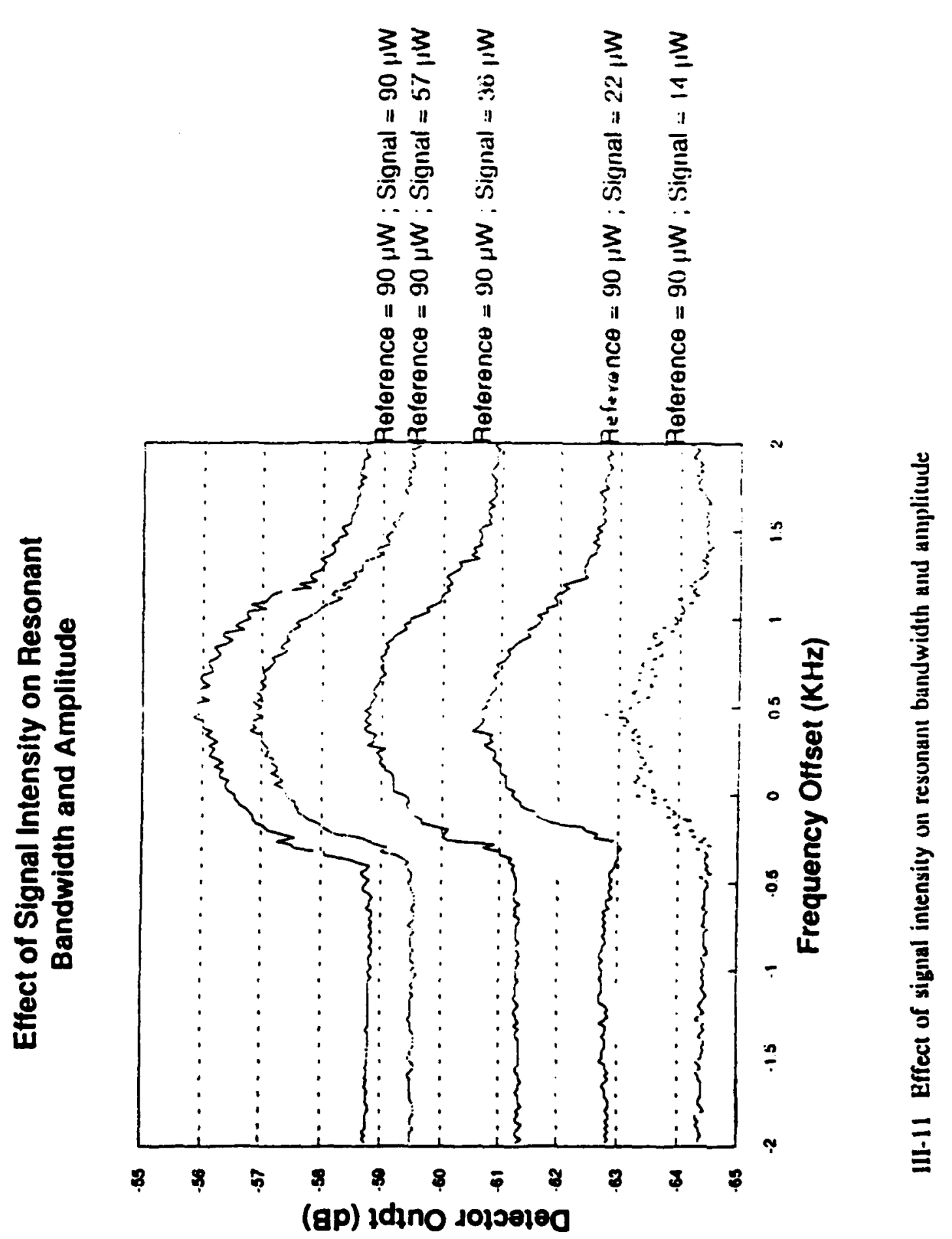




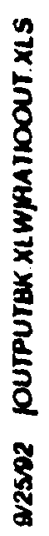

옹

0
퉁
$3 \%$
30

으를

"응 웅

흐 츨 출 흘

边 导

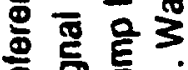

ष的这

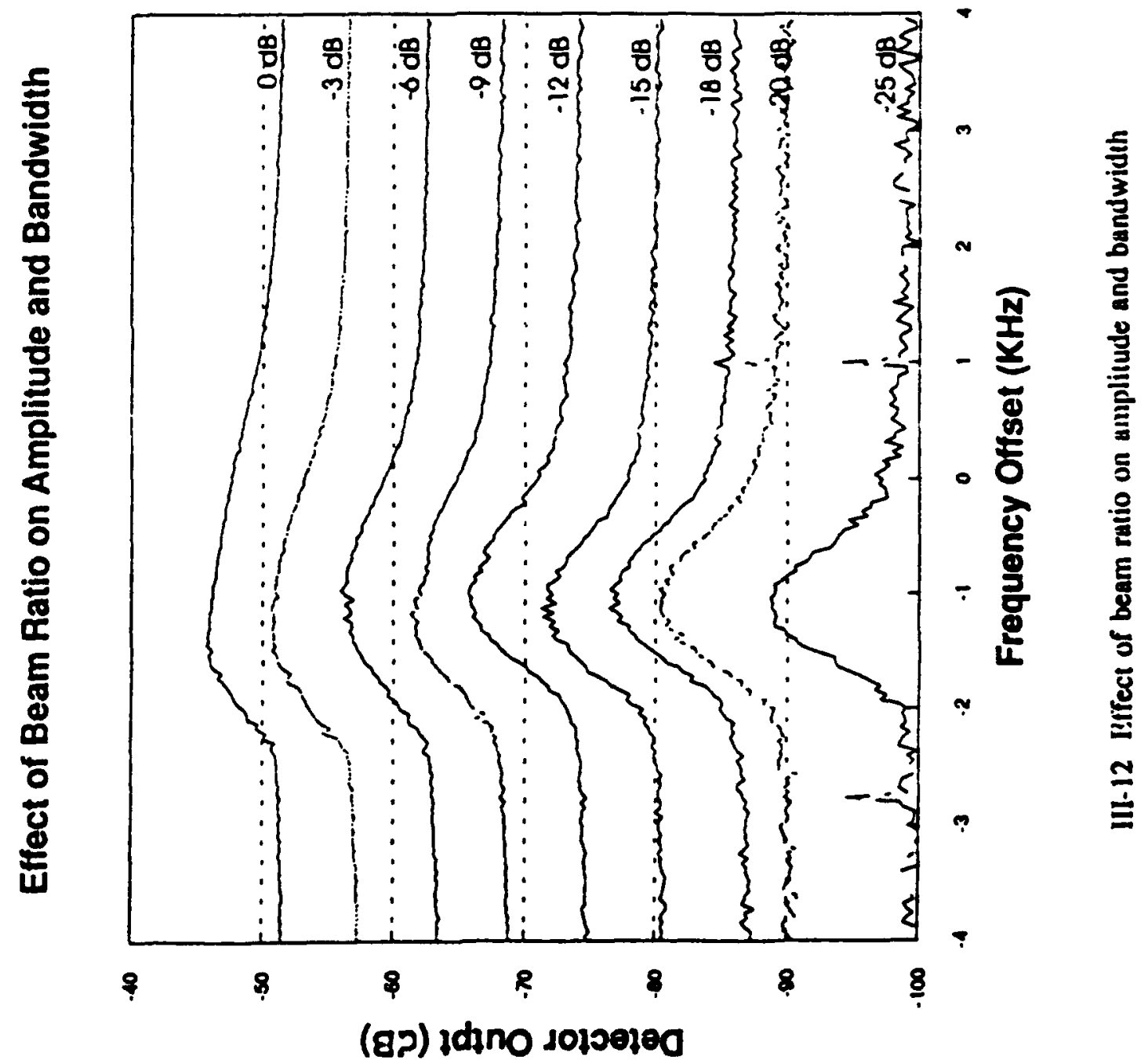


The data in Figure III-12 shows $50 \mathrm{~dB}$ reduction in detector signal for $25 \mathrm{~dB}$ reduction in input beam power. This is as the model of Section IV predicts. Notice the bandwidth broadening at the upper light levels where the incident beam contributes significantly to the photoconductivity. It should be noted that there is a $10 x$ demagnification of the signal and reference so that the 200 microWatts of power corresponds to a $15 \%$ light level increase on the photorefractive material.

\section{Absorption coefficient}

The absorption coefficient partly manifests itself in the governing equations through the Maxwell relaxation time, $\tau_{\mathbf{x}}$. Absorption contributes to photoconductivity in that a percentage of photons absorbed contribute to the photoconductivity (hopefully most of them). The rate of photoelectron generation is approximately given by,

$$
\text { (a) } \frac{d n}{d t}=\frac{a I \eta}{h v}
$$

where $\alpha$ is the optical power absorption coefficient, $I$ is the optical intensity and $\eta_{q}$ is the photoelectron quantum efficiency.

We therefore expect to see trends related to absorption similar to pump intensity. The resonant velocity, inversely related to $\tau_{x}$, should increase as the absorption increases, and the bandwidth should increase as well. Figure III-13 is a plot of two curves taken with the same parameters, but different crystal samples. The absorption coefficient at 1.06 microns of the lower trace is $2.9 \mathrm{~cm}^{-1}$, while the upper trace is $0.62 \mathrm{~cm}^{-1}$.

\section{Vibration correction}

The compensation of system instabilities, such as platform vibration or dynamic atmospheric aberration, is one of the photorefractive processes most advantageous properties. The dynamic Fusing formed in the crystal automatically corrects for wavefront differences between the pump therence beams. The speed at which the grating can adjust is

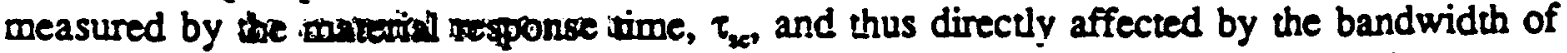
ite signal vs. velocity sosonmoe peak. We would therefore expect to see vibration

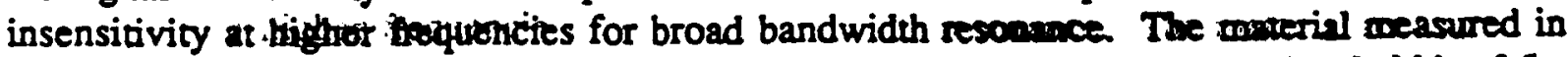

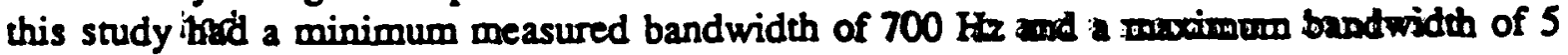
KHz.

Vibration was simulated in the test setup thy placing a piezo translator on one of the folding mirrors in the reference and signal path and driving it with a sinusoidal signal to 
seate a vibrational movement on the mirror. The high voitage amplifier that trove the piezo had a peak drive frequency of about $600 \mathrm{~Hz}$ before it began creating large amour.ts of harxonics in ine drive signal. Measurements above ihis irequency wers not conside:ed weil established due to the higher harmonic components.

The Figure III-14(3) shows a frequency scan as described previously of the naterial sample with no vibration in the system. From the curve we can measure a $3 \mathrm{~dB}$ jandwidth of approximately $800 \mathrm{WHHz}$. The vibration was then added into the system at increasing frequencies, while the peak signal intensity was recorded for each frequency. Figure III-1+(b) shows the results. Although we could not measure out to the full $3 \mathrm{~dB}$ bandwidth with our serup, we can demonstrate that only a small amount of signal strengh is lost due :o vibrations up to $600 \mathrm{~Hz}$. This corresponded well to the frequency scanned measurements for the same experimental conditions of minimum bandwidth. Thus, predictions out to $4 \mathrm{kHz}$ :on the irequency scan should be valid.

\section{Experiment summary}

We have discussed the effects of varying many individual parameters on the grating amplinde, bandwidth and resonant velocity. These effects can be optimized for a system's parricular requirements, and in many cases require a trade analysis berween several opposing characteristics. The primary characteristics are adequately described by the single carrier model for the particular experimental parameters used here. Several key points are listed here as summary to the experimental results.

The pump intensity primarily affects the time response of the grating formed in the photorefractive cell. In most applications the reference power will be weak. The optimum pump power is dependent on the need for vibration and aberration correction in a homodyne system, and additionally on the independent laser linewidths in a heterodyne receiver witi independent laser sources. Increased pump power does not offer the advantage of increased signal level as it does in utilizing a conventional beam combiner. This is a major shortcoming and is discussed further in the following section.

The optical design of the photorefractive system should optimize the angle between the pump and reference beams at the photorefractive crystal, determined by the bandwidth required and the gain of the photorefractive system needed. The gain of the system is only weakly dependent on the beam angles, and thus broad band in an angular sense.

We utilized two modulation methods with the experimental setup. A simple singite sideband modulation with sideband of equal or less strength compared to the carrier intensity and a carrier suppressed single sideband modularion scheme where the carrier was of less intensity than the sideband were simulated by varying the relative intensities of the reference and signal beams. Double sideband modulation was not considered, but results may be extrapolated from the single sideband modulation results. As one might expect, the gain of the system is not a function of signal intensity since this beam is "incoherent" with the other 

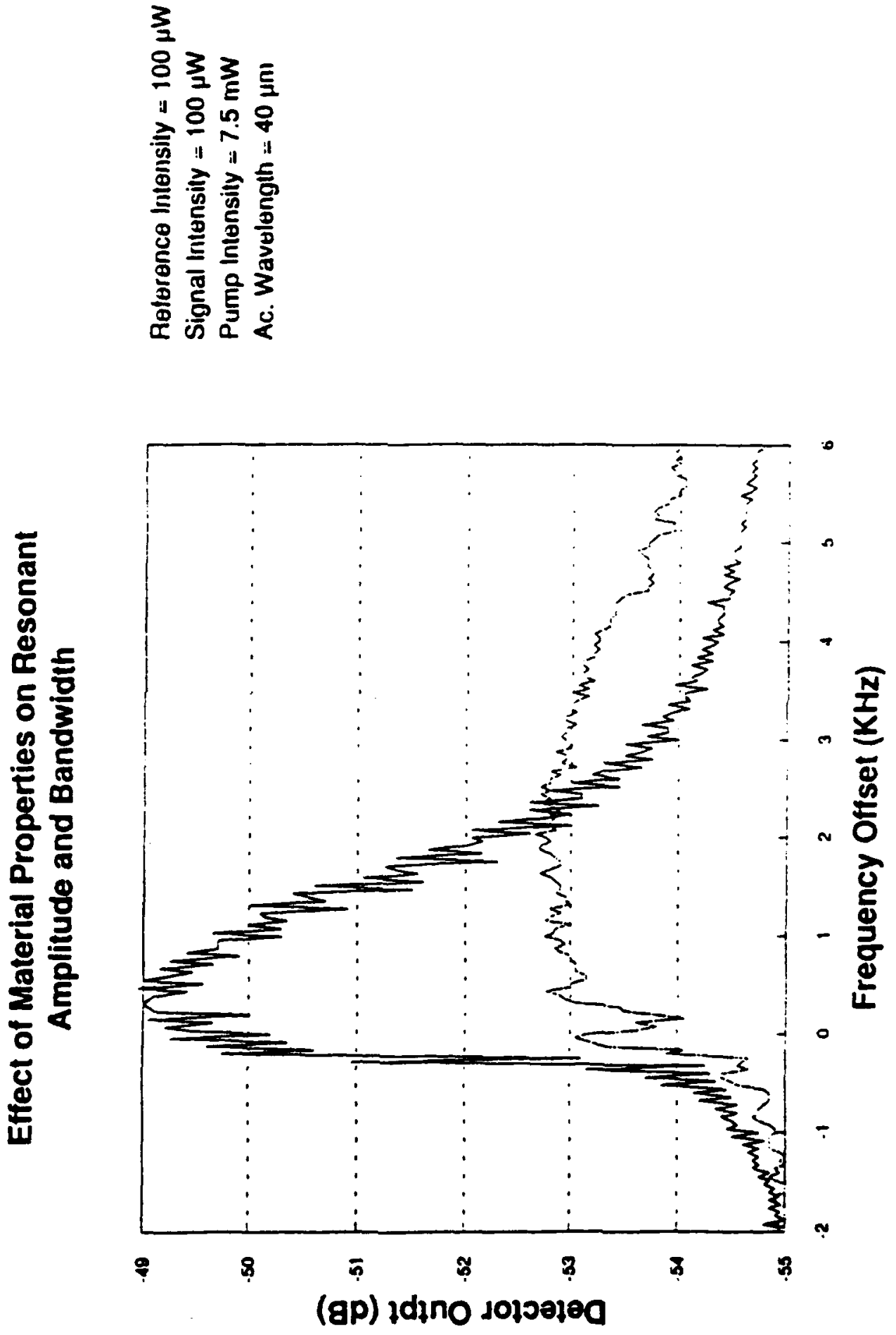

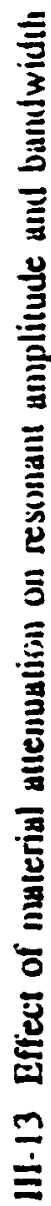




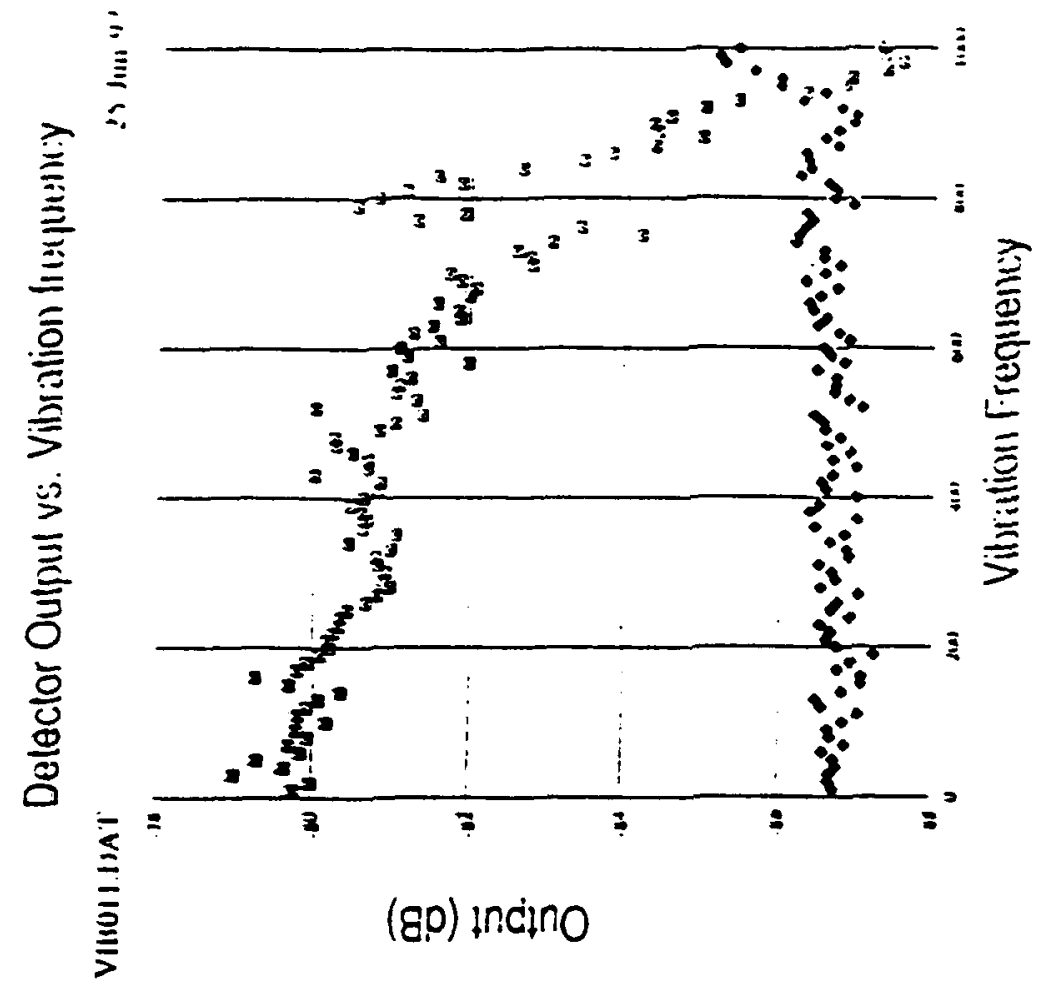


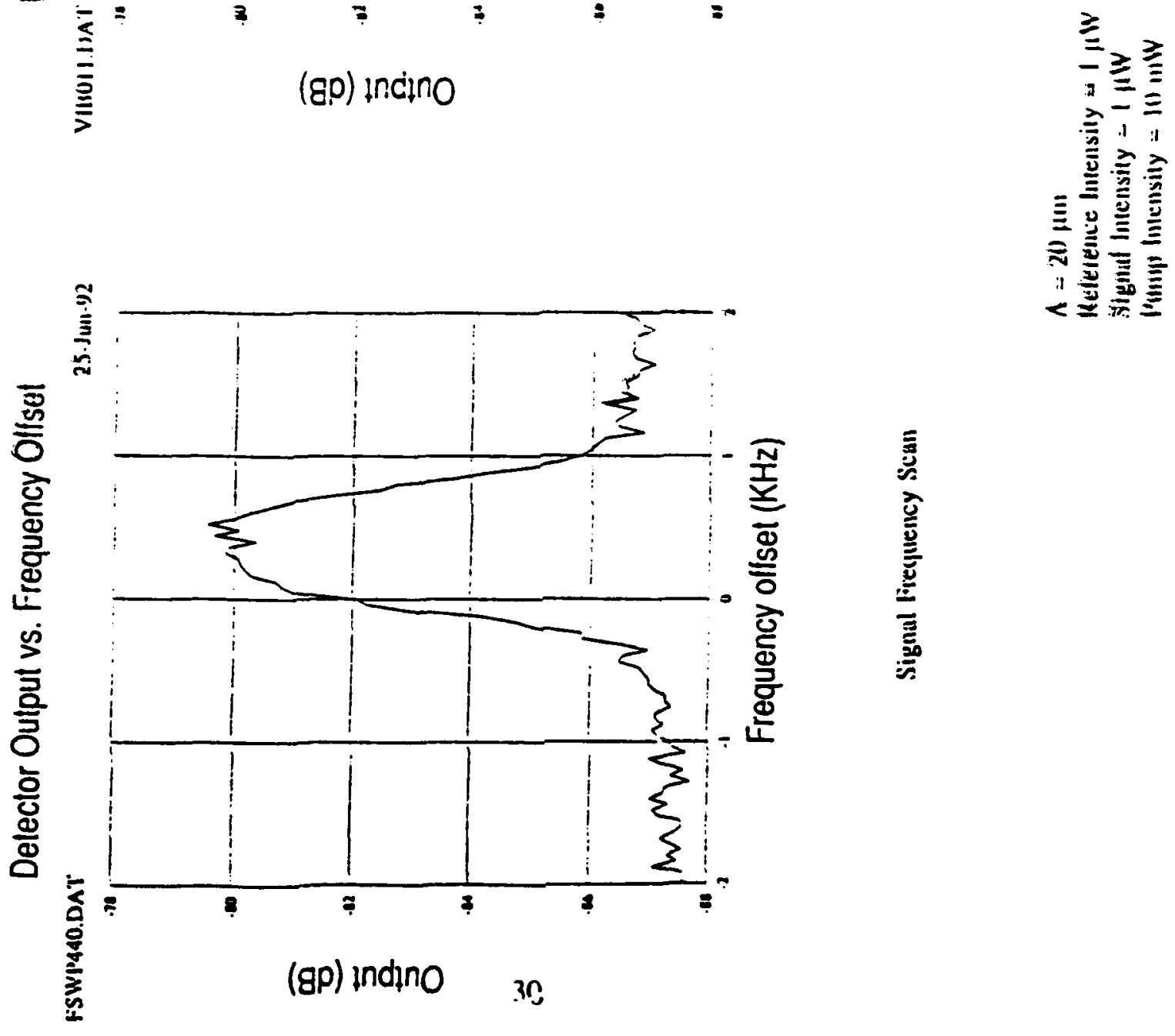
ito beams in the sense that it does not contribute to the formation of the photoreriacjive grating. The signal beam does however contribute to the incoherent erasure of the grating, and may impact the performance of the system if made too large. The graing strength is 1 function of the reference intensity, in the form of the pump and reference intensity beam ratio.

\section{Heterodyne Gain}

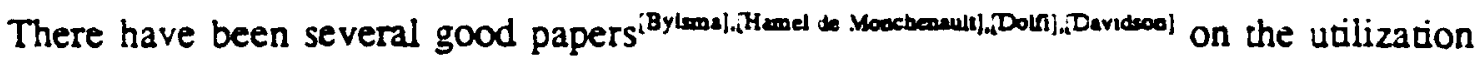
of a photorefractive beam combiner for homodyne/heterodyne signal reception. They have illustrated without doubt that the photorefractive beam combiner works in the sense that signal magnituce can be increased br wavefrs it matched combination of the incident signal with a larger local oscillator. These $k s$ illustrate differential gain of the incident signal and derive relationships to express the $s$ in in terms of photorefractive gain. Application to both digital and analog modulation are shown. Dolfi et. al., discussed the issue of signal-tonoise performance. In the case of Davidson et. al., calcularions and measurements of Bit Error Rate are made for optimum modulation schemes and compared to more conventional optical receiver techniques.

This curent work investigates the requirements on the photorefractive beam combiner, in a general receiver process, for various systems applicability. In particular concentration is placed on the development of simple relationships to perform parameter trades. The issues of interest are heterodyne gain, signal-to-noise ratio (SNR), photorefractive gain and photorefractive bandwidth. These parameters are discussed in terms of their interrelationships.

Heterodyne gain refers to the signal gain due to heterodyne detection of the signal. This is quite apar from photorefractive gain, which is a propery of the photorefractive interaction. Heterodyne gain is perhaps the more appropriate perspective for discussion of heterodyne receivers since it is a system parameter of interest.

Heterodyne gain can occur independent of photorefractive gain. A half coated mirror utilized as a beam combiner will provide heterodyne gain on a photodetector, as will a photorefractive material that has the wrong phase relationship to exhibit photorefractive gain. Photorefractive gain is required, however, for a photorefractive heterodyne receiver system to offer a significant amount of heterodyne gain.

Photoretractive diffraction efficiency is limited to four times the ratio $M_{0}$ of the weak incident reference beam to the strong pump beam for the case in which there is no photorefractive gain, as illustrated in the well known Equation (12) (1)utherever]. In an optical heterodyne receiver this beam ratio is necessarily very small (if not, there is little need for a heterodyne receiver). Diffraction efficiency must necessarily be significantly larger than this limit to offer optimum heterodyne signal gain. It must typically be tens of percent to 
optimize the receiver by diffracting the largest possible local oscillatcr pump onto ine photodetector with minimal loss of the signal. This level of diffracion efificiency san be achieved via the exponential growth in the diffraction graxing stengh produced ur.de: gin conditions. The diffraction efficiency, in the case of gain, car approach $100 \%$ as 11 stes:ec in Equation (13; xakseren! Thus, photorefractive gain is required for small beam rarios to produce significani diffracion coupling efficiencies.

$$
\begin{gathered}
\eta=4 \frac{M_{0}}{\left(1-M_{0}\right)^{2}} \sin ^{2}(\gamma L / 2) \\
\eta=\frac{M_{0}\left(e^{\Gamma L / 2}-1\right)^{2}}{\left(1+M_{0}\right)\left(1+M_{0} e^{\Gamma L}\right)}
\end{gathered}
$$

An experimental measurement of heterodyne gain may be done by a direct comparison of the heterodyae signal, when the photorefractive material is active, to the direct detected signal, when the material is not active (when the pump is blocked). This method of conducting the experiments adds the photorefractive attenuation on top of the direct detect signal, so that for a fair comparison the signal attenuated due to the photorefracive material must be added back on to the direct detect component. That is, the photorefractive material would not be in the light path in a direct detect system, whereas it is in the light path for the heterodyne system. The analysis below presents heterodyne gain as the heterodyne signal detected, compared with the direct signal without attenuation.

In addition to heterodyne gain, a significant parameter of interest, from a systems point of view, is the Signal-to-Noise-Ratio (SNR) of the receiver. In the context of a photorefractive heterodyne receiver the important merit parameter would be the ratio of the heterodyne SNR to the direct detect SNR. Ideally, a receiver system would be shot noise limited in order to maximize the dynamic range. Note that the ratio of signals alone (heterodyne gain) is equal to the SNR ratio when the heterodyne signal and the direct detect signal have a common receiver noise floor, such as thermal noise. 


\section{Heterodyne gain model}

Some simple relationships can be developed which illustrate the dependerce of heterodyne gain un incident light and material parameters in various limits. The relacionships consist of a ratio of the heterodyne signal-to-noise level to the direct detect level, in various noise limits. The model of the problem consists of light incident on the photorefracive material comprised of a pump beam and a low level reference beam that has a modulated signal component. The pump bearn and the reference are coherent or nearly coherent ( $w$ ithin the response time of the photorefractive material) with each other and the signal component is offset in frequency (outside of the material response ime).

The signal may be generated by amplitude modulation of the reference, frequency modulation or addition of a separate beam at an offset frequency (single sideband modulation). The pump and the reference are actually responsible for the generation of the photorefractive grating. Figure IV-1 illustrates the point that regardless of the modulation technique, the unmodulated carrier component can act as the reference. For example, amplitude modulation consists of a dc component with double sidebands of relative magnitude $\mathrm{m} / 2$ each. It is the unmodulated dc component which may be used to interfere with the pump in the photorefractive material to form the grating, while the modulated sidebands simply deflect off of the grating to interfere with the pump on the photodetector. This is illustrated in Figure IV-2, which shows the general receiver concept, where $S_{0}$ is the unmodulated component and $S(t)$ is the temporally modulated part. It is, thus, no great surprise that one can form a photorefractive grating independent of modulation frequency.

A heterodyne signal current is generated on the photodetector when the pump and signal are combine via the photorefractive grating formed by the pump and reference. This current signal has the form.

$$
i_{1+}=\Omega A\left(m S^{2}+P S m \sqrt{\eta}\right) \sqrt{1-\eta} e^{-\alpha L}
$$

This can be compared to the direct detected signal which is observed if the photorefractive element is not present.

$$
i_{s i p}=\Omega A m S^{2}
$$


MODULATION TECHNIQUES

Single sideband
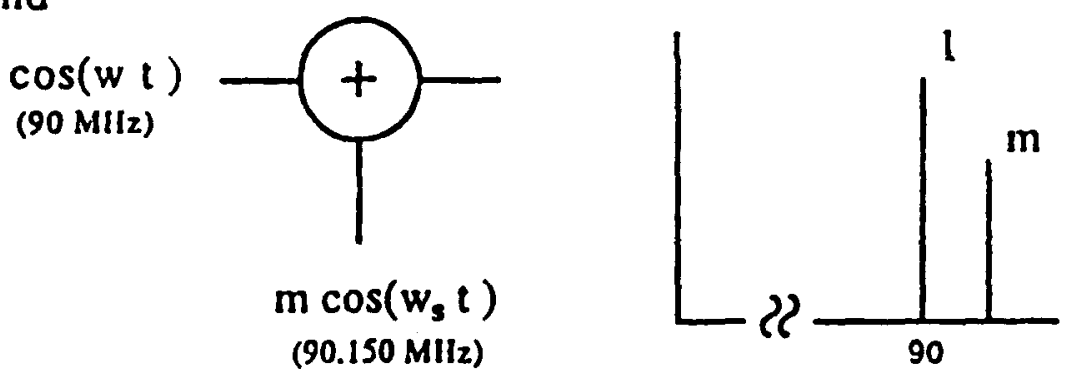

Double sideband AM
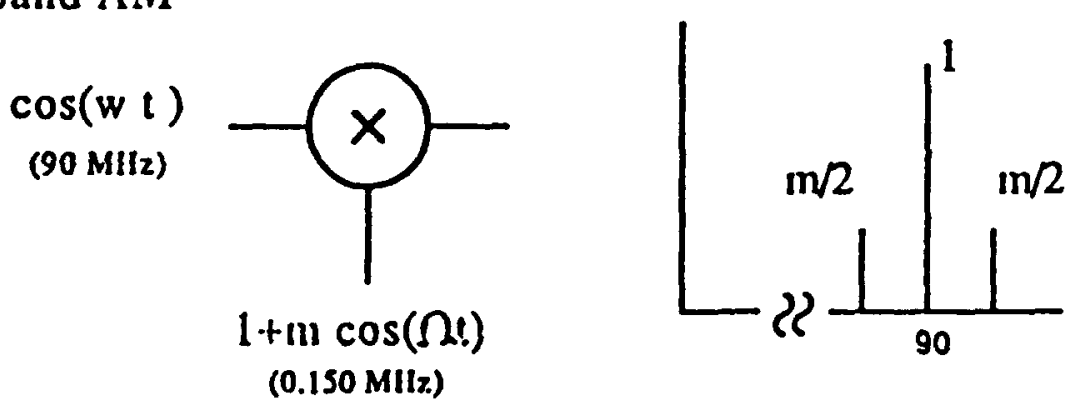

(0.150 Milz)

IV-1 Modulation techniques 


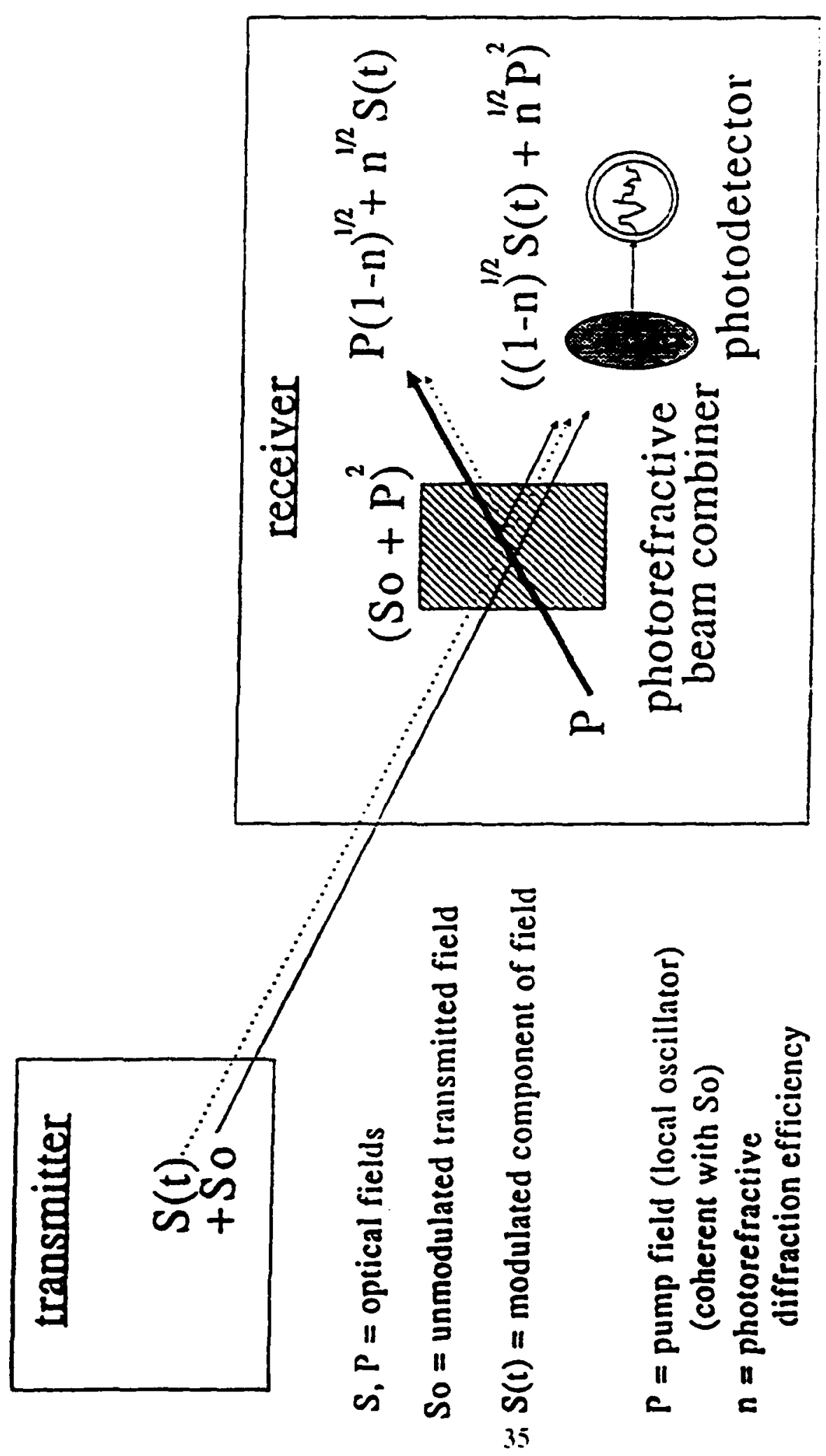

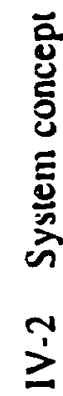


Here, $\Re A$ is the usual detector responsivity end(hv) imes the area ard $m$ is the mocilacor. rato. It represents the ratio of the modulated field component io the unmodulated tc (zeference) field $S$ is the reference field, and $P$ is the pump field it is the light direzeicn exiciency and $x$ is ine optical attenuation coefficient, with $L$ being the optical inte:acscr. iengh.

A ratio of these signals in terns of photoretec:or current is given by.

$$
\frac{S i g_{\text {hor }}}{S i g_{d r}}=\left[1+\sqrt{\frac{\eta}{M_{0}}}\right] \sqrt{(1-\eta)} e^{-a L}
$$

where $I_{b}$ is the ratio of the reference light intensity to the pump intensity. Note that this relationship corresponds to the ratio of heterodyne to direct SNR when detection is dominated by $4 \mathrm{~K}_{\mathrm{B}} T \mathrm{~T} / \mathrm{R}$ noise, the usual thermal noise. It is easily seen that for a system to have a heterodyne gain greater than 1 ,

$$
\frac{e^{\alpha L}}{\sqrt{(1-\eta)}}-1<\sqrt{\frac{\eta}{\beta}}
$$

which requires that the photorefractive gain coefficient must exceed the attenuation coefficient by more than 3 iactor of two. In particular,

$$
\Gamma>2\left(\alpha-\frac{\ln (\sqrt{1-\eta)}}{L}\right)
$$

A similar relationship can be derived for the ratio of heterodyne SNR to direct detect S.VR in the shor noise limit. This relationship gives further insight into the trade-off of receiver parameters. This relacionship is found to be, 


$$
\frac{s n r_{m a}}{s n r_{m}}=\left(\left[1+\sqrt{\frac{\eta}{\beta}}\right] \sqrt{(1-\eta)} e^{-a-i c}\right.
$$

wise:

$$
8=\sqrt{\frac{1 / 2\left(1-m^{2}\right)}{y}} e^{\frac{s L}{2}}
$$

and

$$
\gamma=\frac{1}{2} \frac{\eta}{\beta}+\sqrt{\frac{\eta}{\beta}}+\frac{1}{2}\left(1+m^{2}(1-\eta)\right)
$$

These additional terms result from the shot noise component of the teams.

Relation (19) provides interesting insight into the interrelationship of the light leve!s, modulation depth and diffraction efficiency, but it is not a very practical limit. If the direct detect component were shot noise limited there would be no need for heterodyne detection. Ideally one should retain all of the relevant noise terms and optimize the system parameters to obtain shot noise limited performance of the heterodyne component. Although this approach is appropriate, it leads to more complicated relationships and requires specific assumptions about the system. The simpler relationships above illustrate the relevant trades without requiring specific assumptions about the detector.

In general, the dominant noise terms at signal frequencies are thermal and shot noise. An approximation to the RMS noise current within a given bandwidth is shown in Equacion (22). 


$$
i_{\text {aw }}=\sqrt{\frac{4 K_{\ell} T \Delta f}{R}+2 e \Delta f \Omega A\left[\eta \frac{P^{2}}{2}-\gamma \bar{\eta} P S-\frac{S^{2}}{2}\left(1+m^{2}(1-\eta)\right)\right] e^{-\alpha L}}
$$

The noise current terms illustrated here are the thermal noise and the shot noise terms. The photorefracive noise (beam fanning) and other potential noise terms are assumed small, but could be added as required. Note that the attenuation affects shot noise as the square root of the exponential whereas it affects the signal as the exponential.

The impiications of these relacionships $(16-19)$ are quite interesting. Equation (:7) illustrates that the attenuation can be the dominant term. The fact that attenuation is important is not surprising. It is clear from these relations, though, that the magnirude of the eifect justifies special emphasis. $\eta$ is proportional to $e^{\pi}$ and the detected signal is proportional to the square root of $\eta$, so, as mentioned above, the gain must exceed the attenuation by a factor of two before a positive signal ratio exists. Comparison to experimental data from the literature ${ }^{\text {Talk }}{ }^{-11}$ illustrates this point quite well.

Two particularly interesting cases are the data of Ozkul et. al., and Partovi et al. The data of Parovi et. al. is the highest gain coefficient measured to date in gallium arsenide as a photorefractive material. The data of Partovi et. al. utilizes a short wavelength to obtain band edge gain enhancement, and the Ozkul et. al. data utilizes a long wavelength to avoid high attenuation, as does the data of Bylsma et al. None of the experiments at 1.064 micron YAG wavelengths illustrate significant heterodyne gain performance. The relevant parameters are listed in the following table.

Table IV-1. Gain Comparison

\begin{tabular}{|c|c|c|c|c|c|}
\hline Material & $\begin{array}{l}\text { Gain Coetf. } \\
c \mathbb{m}^{-1}\end{array}$ & Tot. Gain & $\begin{array}{l}\text { Atten. } \\
\mathrm{cm}^{-1}\end{array}$ & Beam Racio & $\begin{array}{l}\text { He: gain } \\
\mathrm{dB}\end{array}$ \\
\hline GaAs ${ }^{\text {[Purcovl] }}$ & 16.3 & 600 & 3 & $1 / 4830$ & 14 \\
\hline 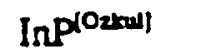 & 6.5 & 100 & 2.6 & $1 / 8000$ & 3 \\
\hline In $P^{\text {(OzzkU] }}$ & 5.3 & 45 & 0.5 & $1 / 700$ & 13 \\
\hline In $\mathrm{P}^{\text {Meningum }}$ & 4 & 5 & 2 & $1 / 80$ & 0 \\
\hline $\left.\ln P^{(B y}(B) a\right)$ & 2.5 & 10 & 0.15 & $1 / 50$ & 8 \\
\hline
\end{tabular}

The calculated heterodyne gain implied from Equation (16) is listed in the last column of the table for all of the experiments. Note that only authors of the last experiment in the table acrually injected signal into the system for measurement, so the results are calculated based on the assumption that the addition of the modulated component does not affect the above measurements. The signal component would have the effect of background light since its frequency offset is outside of the response of the material. This component is typically small compared to the pump beam, however, and should not be significant. 
Vote that the highest gain coefficient provides the highest total gain, and with relatively little loss. This crystal provides the highest heterodyne gain. The second sase illustrates :wice the gain of the third case and an order of magnitude higher total gain thas the fifth. but $\mathrm{i} 0 \mathrm{~dB}$ and $5 \mathrm{~dB}$ less heterodyne gain, respectively. The fourth case demonstrates no heterodyne gain at ail. This clearly illustrates the sensitivity to optical atrenuation. These results follow directly from Equation (17). Another way of looking at this is to say that heterodyne gain is proportional to the pump field incident on the detector while loss affects the combined power since both the pump and signal beam are attenuated.

The curves shown in Figure IV-3 illustrates the signal ratio of heterodyne to direct detect. The curves are plotted against gain coefficient $\Gamma$. $\Gamma$ is related to $\eta$ via the Equation (13). The reference/pump beam ratio $M_{0}$ is shown, three different values, on the curves. The parameters used in Figure IV-3 are from the first experiment ${ }^{\text {Pmovil }}$ in Tabie IV-1, where the curves terminate on the maximum gain coefficient achieved in the experiment. Figure IV -4 illustrates the thermal noise and shot noise limit in the same experiment for an assumed 1 $\mathbf{W H z}$ receiver bandwidth at room temperature. This curve illustrates that there is no advantage of additional gain once the shot noise limit is achieved.

The curves of Figure IV-3 illustrate a diffraction depletion effect versus beam ratio. At small beam ratios a large diffraction efficiency is achieved at moderate gain coeticicients and the heterodyne gain decreases with increased gain coefficient. More diffraction efficiency merely serves to steal the signal beam from the receiver path, as illustrated in Equation (16). For beam ratios less than an $\mathrm{M}_{0}$ of approximately $10^{-3}$, no further significant signal gain is realized at a given gain coefficient. That is, heterodyne gain is independent of pump beam intensity for small beam ratios. This point is also illustrated in Equation (16) by noting that diffraction efficiency $\eta$ is proportional to $\mathbf{M}_{0}$ in the small diffraction limit.

Figure $\Gamma^{-5}$ illustrates the same parameters plotted in terms of the shot noise limited performance, Equation (19). These curves further illustrate the point that if enough signal exists for the direct detection to be shot noise limited, there can be no advantage to heterodyne signal detection. The signal-to-noise is degraded by the heterodyne process even though there may be significant differential signal gain.

\section{Photorefractive gain and gain-bandwidth}

Photorefractive gain is generated when the preliminary photorefractive grating forming maintains a specific phase relationship to the incident light. There are many clever ways to obtain this relationship which has been investigated by many authors (see Table II-1). The gain, ulimately is a function of the material properties and the conditions of illumination. Maximum gain in the traditional photorefractive effect requires techniques to produce the maximum charge separation at a given spatial frequency with small initial light modulation depth by utilizing constructive feedback in the diffracted light interference. Optimum material coefficients produce larger gain coefficients. These include; as large of optical index and electro-optic coefficient as possible, and as small a dielectric coefficient as possible. This 


\section{Heterodyne Gain}

\section{Gain $=20 \log [$ Heterodyne Signal/ Direct Signai $]$}

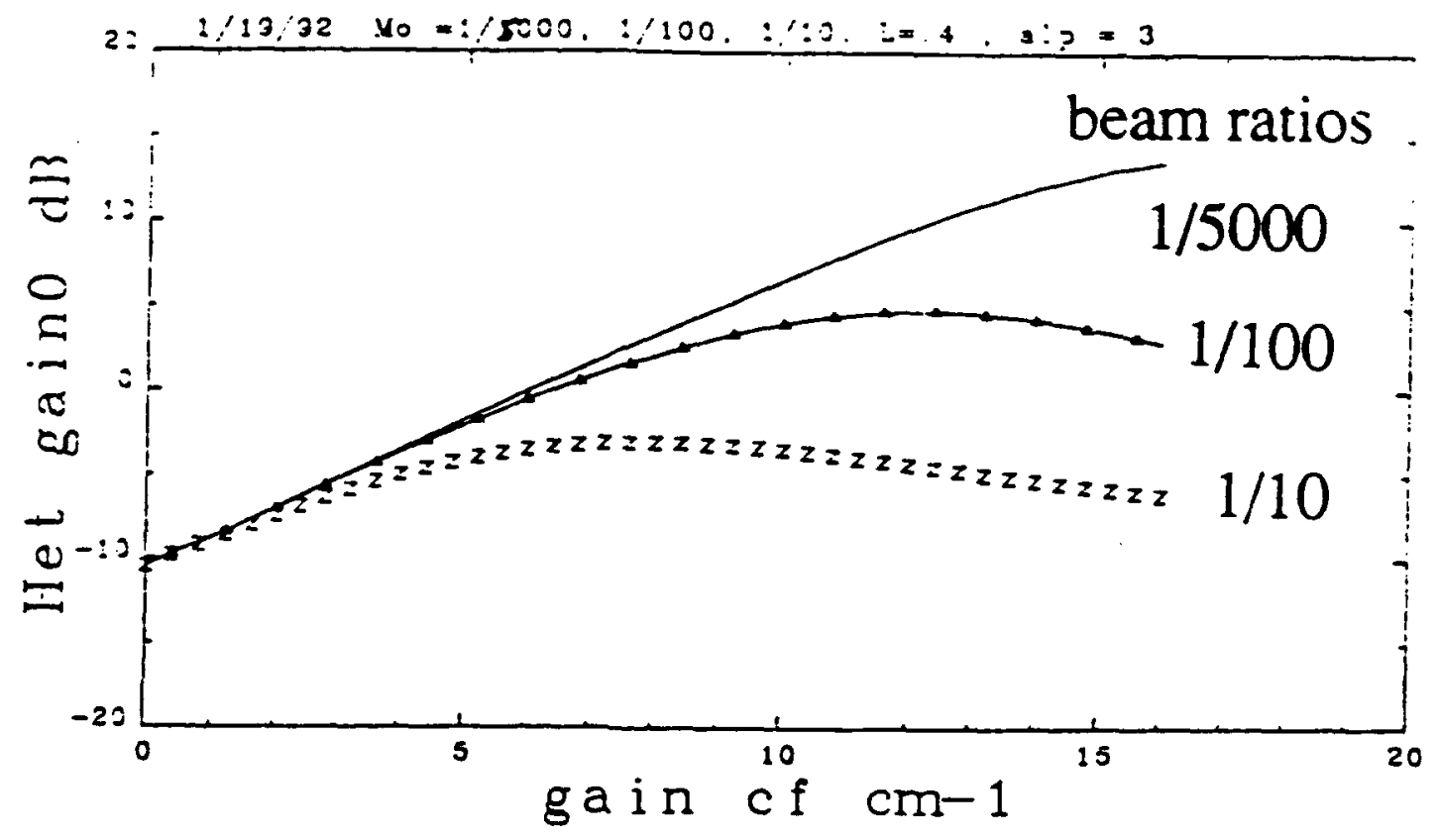

- He: gats: 43

$$
\text { variation with gain coeff }
$$

be:/dir gain 3

\section{IV-3 Heterodyne gain}




\section{Heterodyne SNR}

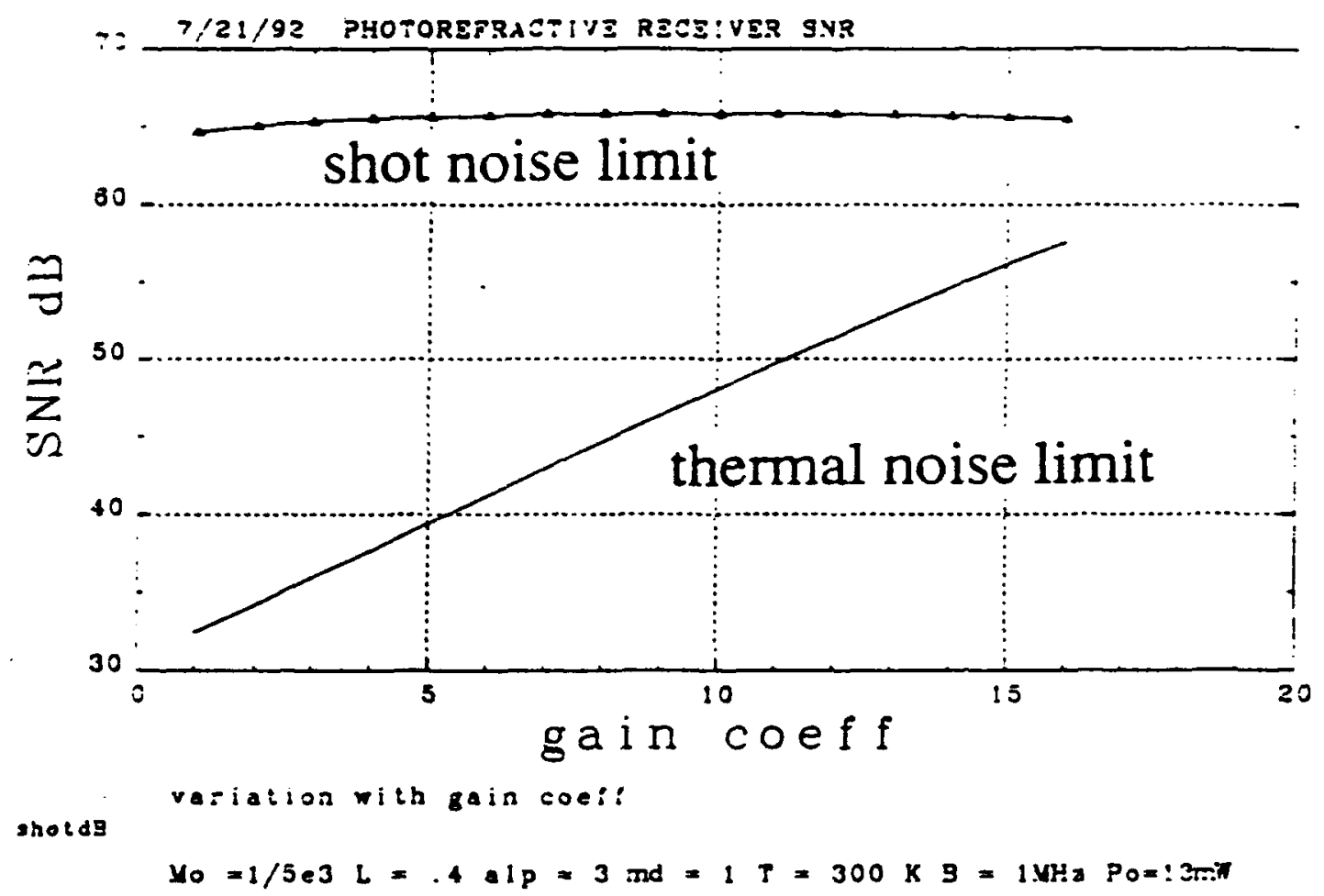

IV-4 Heterodyne SNR 
Heterodyne SNR Gain

Gain $=20 \log [$ Heterodyne SNR/ Direct SNR $]$

$-2 \quad 7 / 21 / 92 \quad Y_{0}=1 / 5000,1,: 00,1 / 10, L=4, \quad .: ?=3$

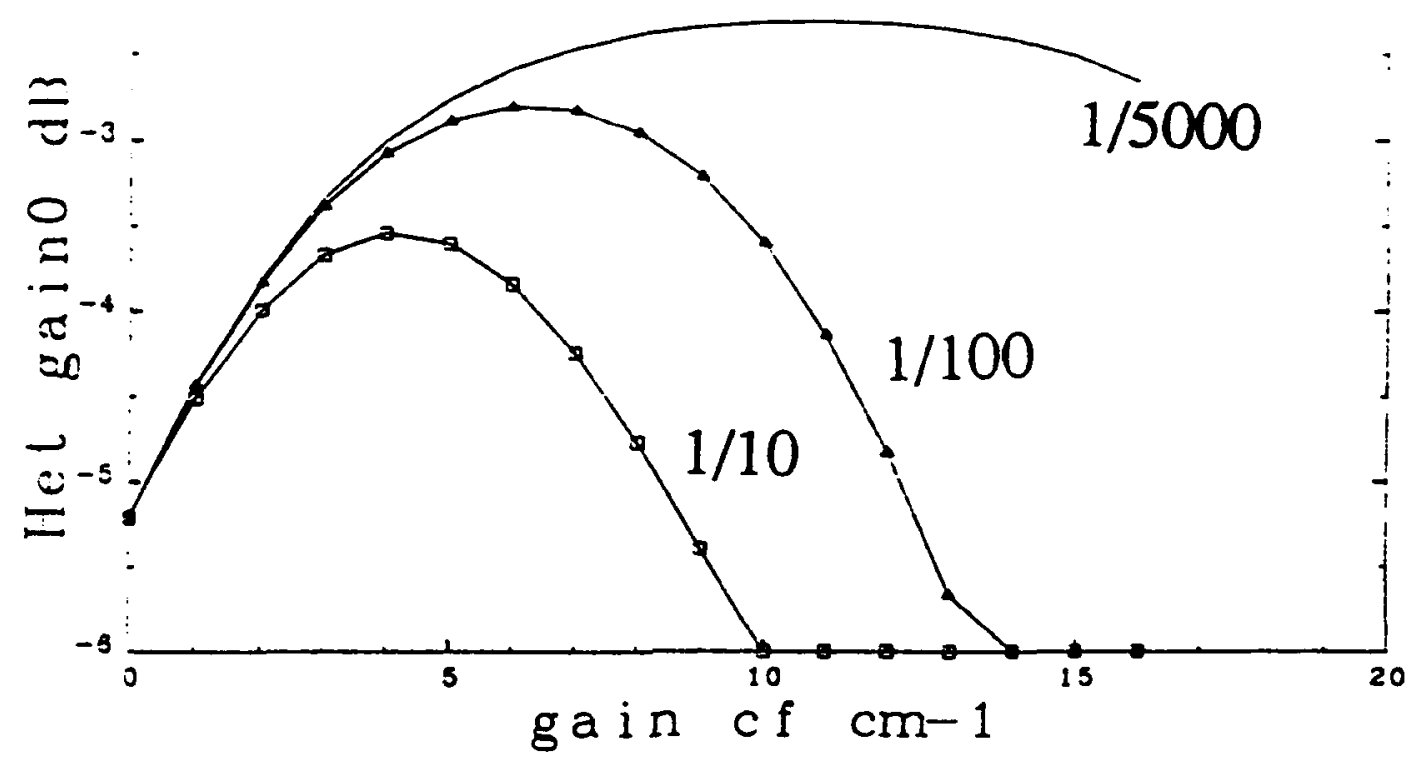

- Het galaz iz varta:ion with gain coe?!

- Het gaial da

hetsing/dirsng and $=1$

IV-5 Heterodyne gain, shot noise limited 
is seen from the iact that the space charge field that forms is proportional to the enirge density $p$ reduced by the dielectric coefficient and the spatiai irequency, or

$$
E_{x} \propto \frac{\rho}{e K}
$$

When substituted into Equation (i) for the diffraction efficiency the basic material parameters contributing to gain become apparent.

The charge density modulation that can be created and maintained is the key parameter that involves the charge dynamic properties of the material. The rate at which a given charge density distribution can be created limits the time response of the material. Gain and time response are competitive processes as illustrated by ${ }^{\text {Yet! }}$,

$$
\tau=\left(\frac{h v}{e}\right)\left(\frac{\lambda}{\Lambda}\right)\left(\frac{\Gamma}{Q}\right)\left(\frac{2}{\pi \eta I Q}\right)
$$

where $Q=n^{3} r / \varepsilon$ and $n$ is the material optical index, $r$ is the electro-optic coefficient and $\varepsilon$ is the material dielectric coefficient. This relationship may be viewed in terms of a gainbandwidth product, since time response bandwidth is inversely proportional to time response of the photorefractive grating. It is apparent from this that pump intensity can offset the gain in the response time of the material. That is, an increase in gain can be offset by an increase in pump intensity to maintain a given response bandwidth. This relationship holds until tap saturation, or some other saturation process, dominates. Some of the rime responses estimated in Table II-1 are scaled from this relationship.

An ideal gain and bandwidth for a given application can be approximated based on the realization that a total gain on the order of the pump-to-reference beam ratio optimizes the signal received. The required photorefractive response bandwidth is on the order of the bandwidth required to aquire the signal (form the grating). Equation (23) can be written in the form,

$$
\tau=\mathrm{x} \frac{\Gamma}{I}
$$

where $\mathrm{K}$ can be measured for a given material under known conditions and is nearly constant 
over cerain ranges of illumination.

Given an incident signal level, the amount of pump :equired on the detec:or :0 cotain shot noise limitec performance of the signal can be determined. Then a bean ratio of $\beta=$ humples can be determined. The gain coefficient that must be achieved for optimum signal reception can be approximated for a given optical interaction length $\Gamma_{\text {spt }}=(\ln (\beta) / L)$. The pump irtensity required 0 achieve the necessary response bandwidth is then tetermined fom Equation (24).

\section{Heterodyne pump dependencies}

In somparison to a more traditional beam combining technique for an optical heterodyne receiver, a photorefractive beam combiner will probably have to provide sufficient gain to achieve shot noise limited performance to be compecicive. A passive beam combiner with fixed reflectivity can always, in theory, put enough pump power on the detector to bring the signal to shot noise limit. For the tradicional beam combiner, exp(- $\alpha \mathrm{L})$ is essentially one and $\eta$ is replaced by the beam combiner refection. The pump can be increased arbitrarily, without affecting the reflectivity.

In the photorefracive case, the beam combiner deflecion efficiency is given by, in the appropriate limit, $\eta=\mathrm{M}_{0} \mathrm{e}^{\Omega}$. Thus, as purap power is increased, diffraction efficiency is decreased, maintaining a fixed amount of pump power incident on the detector. This is illustrated in Figure IV-6, comparing a conventional fixed reflectivity beam combiner with a photorefractive beam combiner utilizing the parameters from the first experiment in Table IV1. Note that $10 \mathrm{~dB}$ of pump power increase corresponds to $10 \mathrm{~dB}$ of detector signal for the conventional beam combiner, whereas the photorefractive beam combiner signal is independent of pump power once the beam ratio gets above the diffraction depletion limit. If insufficient photorefractive net gain exists under the conditions of the illumination, shot noise limited performance cannot be obtained by increase pump power.

The photorefractive beam combiner does offer the advantage of wavefront and jitter compensation. However, if the transmitter laser and local pump are several $\mathrm{kHz}$ line width lasers, the current state-of-the-art, that are frequency locked together, a $\mathrm{kHz}$ of mechanical vibration is inconsequential. In addition, the material response bandwidth would have to be greater than the correlated laser linewidths to develop a diffracrion graning. If wavefront aberration is a problem on the incident light a smaller aperture can be sampled and mixed with larger local pump power utilizing a conventional beam combiner which is designed with optimum reflectivity. The photorefractive beam combiner might accept a larger,

nondiffraction limited, aperture. But it would require one to help compensate for the optical losses. Thus, appiication of currently available photorefractive materials to a remote heterodyne receiver for the purpose of signal-to-noise gain does not appear to offer significant advantage. 


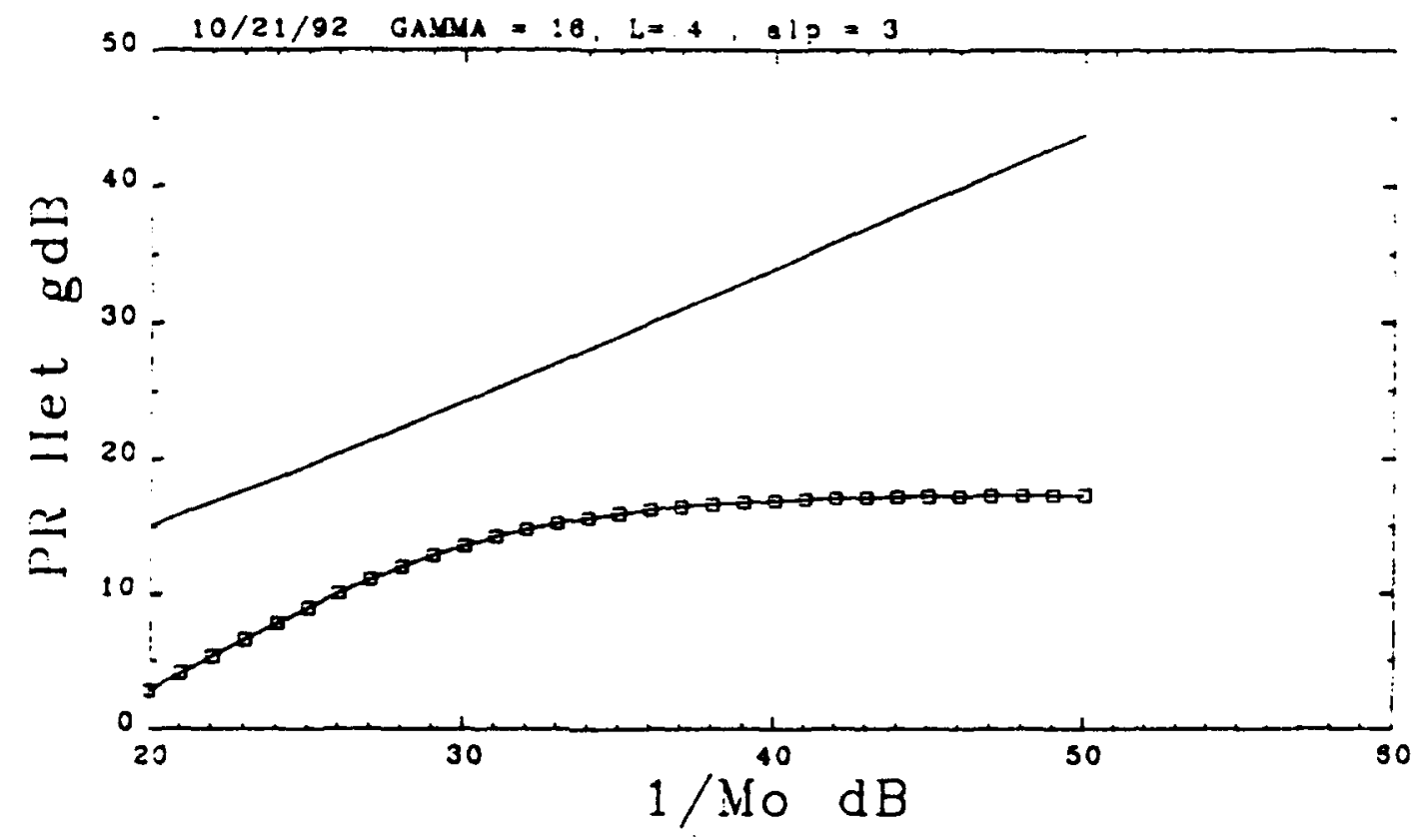

- IL Het gdB

variation with pump Intensity

IV.6 Comparison of conventional to photorefractive beam combiners 
A. opticai homodyne system. which utilizes the sarfe laser as je rierence and pump. nay benerit tom utilizing a photorefiacive material as an acaptive seam combiner. For this case the laser linewidth only need ce sufficiently narow :o sccount :cr sparial pati: differences berween beams. Benefit is derived from the insensivivity :o rechanicai vitracior. within the respense ime of the material and insensitivity to alignment in addition :0 the insensitivity :o guality of the optics. Furhermore, multiple jeams can be combined simultaneously. Wany other unique applicarions have been discussed for photoretracsive eiements as bean combiners including application to phase iocking !asers, image processing and others (see references).

\section{Conclusions and future potential}

The resuits discussed above indicate that based on eirendy aiaijole materias urilization of a ghotoretracjue adaptive beam combining element for heterodyne reception may be limited to a narrow range of special applications. The primary reasons for this may be stated simply as inadequate gain and inadequate response ime of the available raterials for many potencial applications. The explaination is more involved.

From a systems perspective heterodyne gain is a key parameter of interest. Furthermore, he:erodyne gain is only of benefit when the signal floor is limited by fixed receiver noise, such as thermal noise. The advantage of heterodyne gain is to bring the signal up out of the receiver noise, ideally, to its shot noise limit. Photorefractive gain must provide adequate diffracion efficiency at the system's required beam ratio to achieve significant signal gain after suffering the attenuation of the material. It must have adequate response time to accomodaie frequency uncertainties between the signal carrier and the local pump.

In a conventional beam combiner heterodyne system the heterodyne gain is proporitional to the local pump power. In a photorefractive beam combiner it is independent of local pump power. In terms of the beam combiner, for a Exed level of incident signal a conventional beam combiner inherently has a pump-to-signal diffaction gain proportional to the pump power (it has a fixed diffraction efficiency), the photorefractive gain is independent of pump power (its diffraction efficiency varies inversely as pump power). This is a problem if inadequate photorefractive gain exists at the required operating conditions. With measured photorefractive gains typically less than $100 \mathrm{few}$ real receiver systems would derive benefit from a photorefractive beam combiner. This is particularly oue when the optical atrenuation required to form the diffraction grating is considered.

If an iceal photorefractive material were envisioned for this application it would have a response time adequate to develop a diffraction grating under condicions of the correlated laser linewidths of a transmitter laser and local pump laser or compensate for frequency offsets or platform jitter. The material must have adequate gain to compensate for twice the attenuation, just to break even, and it should have sufficient gain to allow the local oscillator to bring the signal up to the shot noise limit of the detector. A survey of results from the literature showed that very few of the fast reponse materials measured offered real heterodyne 
gain, and ones that did had inadequate measured, or estimated time response for a separate transmitter-receiver heterodyne system.

Furure hope for this application of photorefractive materials lies in the development of new materials. One of the most exciting of new materials is the Multiple Quantum Well (MQW) device. These devices are essentially "designed" materials. By alternating very thin layers of semiconductors materials with specific trap states and carrier lifetimes can be designed. This allows the development of very sensitive photorefractive materials at very useful wavelengths such as $800 \mathrm{~nm}$.

The MQW materials have significantly greater (orders of magnitude) sensitivity (gainbandwidth potential) than traditional bulk semiconductors. They offer a great new potential for the field of photorefractives in general. Their primary shorcoming at this point is with regard to applications that require photorefractive gain. The device interaction length is currently limited to tens of microns. Thus, the gain length product is very limited, even if very large gain coefficients are obtained. This is not purely a fabrication limitation. Since the electric field is applied parallel to the light interaction, a long interaction would short out the spatially modulated electric fields. Multiple devices stacked serially in the light interaction direction might be an approach to provide gain length. Another potential shortcoming is the high attenuation required to achieve gain.

MQW devices generally utilize an absorption process to generate the index variation (electro-absorption) in a Kramers-Kronig type relationship. They are, thus, more complicated than the traditional photorefractive effect. They exhibit very sharp exciton absorption lines and as such are very sensitive to wavelength. This additional complexity has significant implications on system applications.

Preliminary performance of these devices has been quite impressive. Partovi et. al. measured $3 \%$ diffraction efficiency in a MQW device at $830 \mathrm{~nm}$ wavelength. Wang et. al., describe some of the recent advances in this area. They achieved nearly $1000 \mathrm{~cm}^{-1}$ gain coefficient in experiments. This is the highest gain ever achieved in any photorefractive device. The absorption on this measurement, however, exceeded the gain. Thus, more development is required. The key point is that no other material offers the potential of these devices, but further investigation is required to assess their viability for a given application. 


\section{REFERENCES}

R. B. Bylsma, A. M. Glass, D. H. Olsen, "Optical Signal Amplification at 1.3 micron by Two-Wave Mixing in InP:Fe", Electr. Lett. 24(6),360,(1988).

F. Davidson, C. Field, X. Sun, "20 Mbps optical homodyne communication system using photorefractive beam combining", Photonics Technology Lett. Nov.,(1992).

D. Dolfi, B. Foretier, B. Loiseax, H. Rajbenbach, JP. Huignard, "Coherent detection of optically carried microwave signals through two-wave mixing in a $\mathrm{BaTiO}_{3}$ crystal", Appl. Optics, 29(35), 5228,(1990).

Duncan, T. H. Liu, L. J. Cheng, "Photorefractive gain in GaAs under a de electric field", Appl. Phys. Lert. 53(15),1369,(1988).

G. Hamel de Monchenaul, J.P. Huignard, "Two-wave mixing with time-modulated signal in $\mathrm{B}_{12} \mathrm{SiO}_{20}$ theory and application to homodyne wave-front detection, J. Appl. Phys. 63(3),624,(1988).

G. Hamel de Monchenault, B. Loiseax, J.P. Huignard, "Amplification of high bandwidth signals through two-wave mixing in photorefractive $\mathrm{Bi}_{12} \mathrm{SiO}_{20}$ crystals", Appl. Phys. Lett. SO(25), 1794,(1987).

B. Imbert, H. Rajbenbach, S. Mallick, J.P. Herriau, J.P. Huignard, "High photorefractive gain in two-beam coupling with moving fringes in GaAs:Cr crystals", Opt. Lett. 13(4),327,(1988).

M.B. Klein, S.W. McCahon, T.F. Boggess, G.C. Valley,"High-accuracy, high-reflectivity phase conjugation at $1.06 \mathrm{uM}$ by four-wave mixing in photorefractive gallium arsenide", J. Opt. Soc. Am. B, 5(12),2467,(1988).

N.V. Kukhtarev, V.B. Markov, S.G. Odulov, M.S. Soskin, V.L. Vinetskii, "Holographic storage in electrooptic crystals. I. Steady state", Ferroelectrics, 22, 949,(1979).

B. Mainguet, "Characterization of the photorefractive effect in InP:Fe by using two-wave mixing under electric fields", Opt. Lett. 13(8),657,(1988).

J. E. Millerd, S. D. Koehler, E. M. Garmire, A Parovi, A. M. Glass, M. B. Klein, "Photorefractive gain enhancement in InP:Fe using band-edge resonance and temperature stabilization", Appl. Phys. Lett. 57(26),2776,(1990).

C. Ozkul, G. Picoli, P. Gravey, J. Le Rouzic, "Energy transfer enhancement in photorefractive InP:Fe crystals using an auxiliary incoherent beam and a negative thermal gradient", Opt. Eng. 30(4),397,(1991). 
C. Ozkul, G. Picoli, P. Gravey, N. Wolffer, "High gain coherent amplification in thermally stabilized InP:Fe crystals under de fields". Appl. Opt. 29(18),2711,(1990)

A.Partovi, A. Kost, E. M. Garmire, "Band-edge photorefractive effect in semiconductors", Appl. Phys. Let. 56(12),1089,(1990).

S.I Stepanov, MP. Petrov, "Nonstationary Holographic Recording for Efficient Amplification and Phase Conjugation", Topics in applied physics, Vol. 61, chapter 9., ats. P Gunter, J.P. Huignard, Springer Verlag, (1988).

Q. Wang, R.M. Brubaker, D.D. Nolte, M.R. Melloch, "Photorefractive quantum weels: transvers Franz-Keldysh geometry", J. Opt Soc. Am. B, Y(9), 1626,(1992).

P. Yeh, "Fundamental limit of the speed of photorefractive effect and its impact on device applications and material research", Appl. Oprics, 26(4),602,(1987). 
adjuesios

nupts $\rightarrow r$

cf cusies

2L/CB..A

5

ITTV: N:TER UUSHUNON

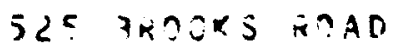

JE:FFIS: IFE UY $134+1-45-5$

HA ORIS CA= DEPATIC:H

GUVERNMEAT AEZTJFACE IYS DIV

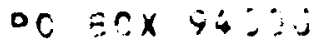

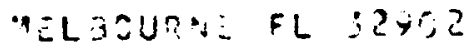

$9 ! 15 \mathrm{UL}$

$T \equiv E H V I C E L$ LESAARYY

So $C ! \equiv T T=0 \because I I C$ PKY

SRTFFIS3 dF सY $13441-4514$

AONINIST:

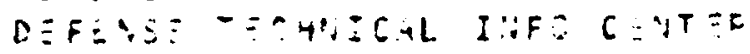

DT: $\{-F D A C$

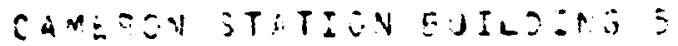

$A L=X 4: 2 \div I 1$ VA $Z Z Z: 4-3145$

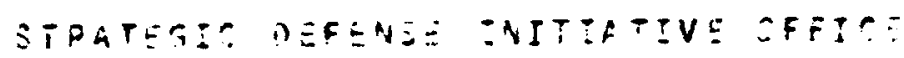

$?$

OFEICE JE THE SECIETAPY OF OEFENSE

WASH or ?:O1-71:-

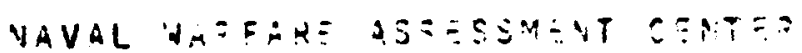

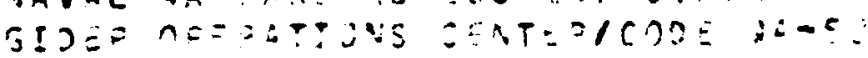

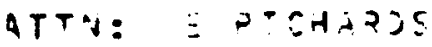

007044 C: $21710-5 \cdots:$

HO AFVITKTH

$\uparrow$

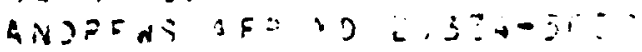

$1 z$ in: $013=I Y$

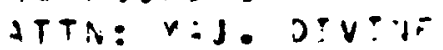

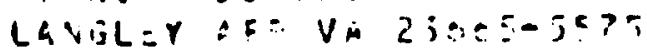




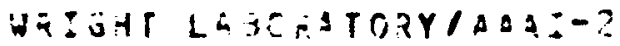

MAXWELL AFE IL $33112-53 C 4^{\circ}$

US ARMI STRATEGIC PEF

CSSD-:Y-D:

PJ JOX 15??

HUNTSVILLEAL 35507-33:1

COMMANOIVG OFFICE

IAVAL AVIJNICS CEMTER

LI TAATYY D/765

INOIANAPOLIS IN 4 E210-219O

COMMANOING JFFICER

NAVAL OCEAN SYSTEMS CENTEP

TECHNICAL LIBOEPY

CODE $9442 B$

SAN D:EF? CA 92152-502r

$$
\text { DL- ? }
$$


CMDR

IAVAL DEAPONS CENTEE

TECHNIG LL LIBRARY/C 3431

CHINA LAKE CA 93555-E I:1

SUOERINTENOENT

CODS 5?4

NAVAL DSSTGRADUATE SEHOOL

MJNTEREY C\& ?394?-5C3J

SPACE : VAVAL WARFARE SYSTEMS COMM WASHIVGTON OC 2C363-51:

CDR, U.S. ARMY MISSILE COMMAND

REDSTOVE SEICITIFIC INFE CENTEA

AMSMI-RD-CS-RIILL DCCUMENTS

FEOSTCVE GFSEVAL AL 35372-5241

ADVISCOY GTOUF ON ELECTEON DEVICES

ATTNE OJCUMENTS

2011 CPYSTIL DPIVE,SUITE 3C7

ARLIVGTSY VA Z22J2

LOS ALAMOS NATIONAL LABAFATOFY REPCDT LTSZAPY

NS $2 \mathrm{CO}^{-}$

LOS ALANIS NM P7544

$A \equiv D C \quad L I S O \& P Y$

$T \equiv C H \quad F I L=S / M S-1 . J S$

ARNOLD AFE TN 37330

COMMANDEZ/USAISC

ATTN: ASJF-OC-TL

SL OG $61: \div 1$

FT HUACHUCA AI $\triangle 5013-5 \%$ IF?

IIR WFITHEO SEOVICF TECHIICAL LIF

FL LS1:

SCOTT AFE IL $02225-5453$

$$
O L-3
$$


SAN ANiLA:? TX 70243-5:?

SOFTHAFE EVGIVEEFINO INST (SEI)

TECHNICAL LITRARY

SC JU F?EZES LVE

PITTSUU:TH PA 13213

DIRECTOR ISAIESS

W157

QB?U SAVAJE PJAD

FCPT MEATE MO 21C55-65CE

VSA

T51221T2L

FODT MEADE MD 20755-6300

NSA

ATTN: D. ALLEY

DIV X911

- 30G SAVAGE FOAD

FT MEADE MO 23755-0.200

000

231

OSOS SAVAGE ?040

FT. YEACE MD 20755-0JUD

TIRNSA

1

?. 529

78TJ SAVAGE POAD

FT $M \equiv A D E$ M 23775

TIRECTIR

1

NSA/CSS

ROS/P $5=4 L D S$

FOPT GEJPSF G. MEADE MD Z7755-62JJ

ESE IC

5O GPIEF:SS STREET

HANSCOM IF WA 01731-1610

$D L=4$ 
$2 J$ SEHILLI:IS CIPCLE

HANSCJY AF $=$ MA $31731-2216$

FL 2PST/RESEAFCH LIERANY

CL AA/SULL

HANSEOA AFA MA S17?1-5L2!

TECHNZEAL PEPOPTS EENTEP

MAIL DPUP D13:

TURLINGTON ROAD

DEDFORD MA O1731

DEFENSE TECHNOLORY SEC IDMTN (DTSA)

ATTM: STTD/PATRICK SULLIVAN

40? ARTY NAVY DRIVE

SUITE ZC:

ARLINGTON VA 222R?

MIT LE:COLY L4SOOAT20:ES

1

ATTII: V.W.S. CHAV

$L \equiv X I N G T O N$ YA $Z 2173-L L 73$

SOIO TIC

ATTIN J JE TILTON

THE DESTAGDN

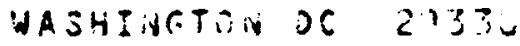

SAF/AJS:

THE DENTAIJON

WASHENGTON OE 2035

$A F S C / C V-0$

ATTN: LTE FLYUN

ANDREW: AF AD ER $334-59 R$

Ha $501: 4: 3$

ATTN: *. LOAGUS

P.). $3: x$ 0256:

WOPLOWAY ONSTAL CENTER

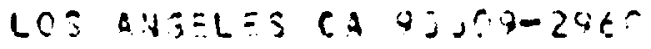


ATTAE LOE OLDEV3EDG

HANSCSM EF = YA 21731-5:2?

AFSTER:AT

ATTN: LTC DETUEE:

KIRTLAVD AFa NB ??117

USA SDCIDASO-HOSB

ATTNE LAPOY TUJES

P.D. POX 1:0?

HUNTSV LLE AL 35:j?

ANSER COPPOPATION

SUITE SU:

CRYSTAL GATEWAY 31

1215 JEFFEPSON DAVIS HIGHWEY

ARLINGTCN VA 22202

SDIOIGYE

THE DENTEGON

WASHENGTO DC 2CES1-T1C:

SOIO/OT:

1

THE PENTAGON

WASHINCTOA DC 2CZ:1-71F?

SOIO/CTS

1

THE DENTAGCN

WASHIVGTON DC 20331-710:

DROGRA EXECUTIVF PFFICEIGPALS

1

$O F \equiv A-G P L-R C C-P D J-C$

D. O. HOX 15C

HUNTSVILLE AL 35907-3301

COMMAR?ING DFFICË

NAVEL FESEAREH LARCRATCOY

COOE 2 KOT

WASHINGTOA OC 20375-5.3E: 
MITRE CJKP

Y/S E25: ATTVE Z.T. CADLSON

BURLINETIN $2 O+D$

BECECRE MA BI730

SDIOIDTE

ATTN: CDR KOFEJWO

THE DENTTAGON

HASHINGTON DC 203J1-710? 


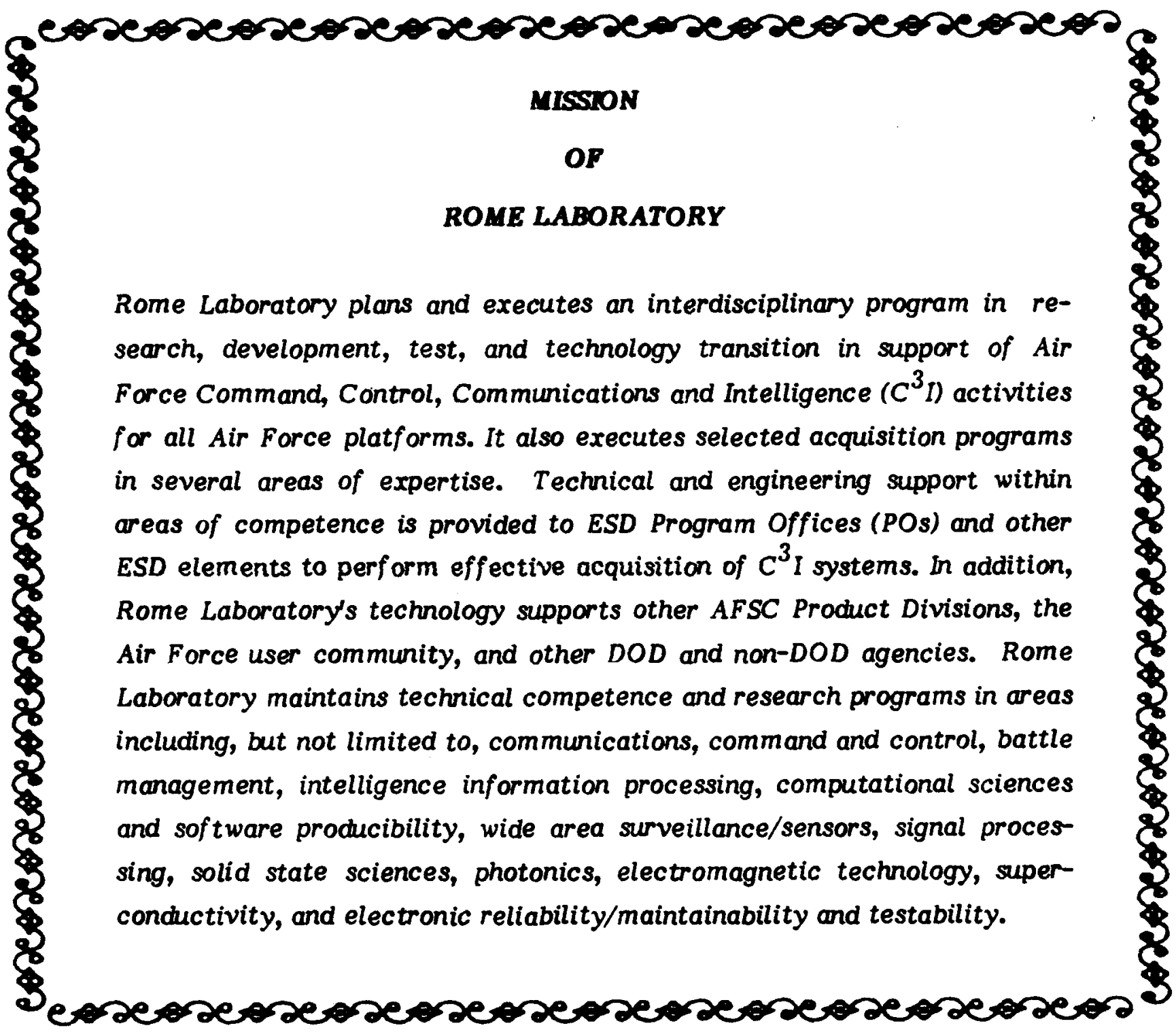

